\title{
Legendre integrators, post-processing and quasiequilibrium
}

\author{
Alexander N. Gorban ${ }^{\mathrm{a}, *}$, Pavel A. Gorban ${ }^{\mathrm{b}}$, Iliya V. Karlin ${ }^{\mathrm{a}}$ \\ a Department of Materials, Institute of Polymer Physics, Swiss Federal Institute of Technology, ETH-Zentrum, \\ Sonneggstrasse 3, ML J 27, CH-8092 Zurich, Switzerland \\ ${ }^{\mathrm{b}}$ Krasnoyarsk State Technical University, Institute of Computational Modeling RAS, Krasnoyarsk 660036, Russia
}

Received 17 September 2003; received in revised form 15 December 2003

This article is part of a Special Volume containing papers from the 3rd International Workshop on Nonequilibrium Thermodynamics and Complex Fluids

\begin{abstract}
A toolbox for the development and reduction of the dynamical models of nonequilibrium systems is presented. The main components of this toolbox are: Legendre integrators, dynamical post-processing, and the thermodynamic projector. The thermodynamic projector is the tool to transform almost any anzatz to a thermodynamically consistent model. The post-processing is the cheapest way to improve the solution obtained by the Legendre integrators. Legendre integrators give the opportunity to solve linear equations instead of nonlinear ones for quasiequilibrium ("maximum entropy", MaxEnt) approximations. The essentially new element of this toolbox, the method of thermodynamic projector, is demonstrated on application to the FENE-P model of polymer kinetic theory. The multi-peak model of polymer dynamics is developed.
\end{abstract}

(C) 2004 Elsevier B.V. All rights reserved.

Keywords: Nonequilibrium systems; Mathematical modeling; Entropy; Thermodynamic projector; Post-processing; Fokker-Planck equation

\section{Introduction}

There are many attempts to fill the gap between the microscopic and the macroscopic models, and to construct closed macroscopic equations. Most of the closure assumptions have a relatively narrow domain of applicability, and their usage has the following problems:

(1) Violation of basic physical (thermodynamics) laws.

(2) Absence of accuracy control procedures.

(3) Absence of a successive step-by-step procedure of the refinement of a model.

The main object of investigation is the evolution equation

$\dot{\Psi}=J(\Psi)$,

where $J$ is some operator and $\Psi$ is the distribution function over the phase space.

The constructed methods are aimed at extracting the dynamics of the macroscopic variables from the microscopic Eq. (1). The prototypes of these methods are the quasiequi-

* Corresponding author. Tel.: +41 163266 57; fax: +41 16321076 .

E-mail addresses: agorban@mat.ethz.ch (A.N. Gorban), pavelgorban@yandex.ru (P.A. Gorban), ikarlin@mat.ethz.ch (I.V. Karlin). librium approximation, dual integrators and the thermodynamic projector.

The quasiequilibrium closure for the set of macroscopic variables $M(\Psi)$ is built with the help of the solution to the variational problem (MaxEnt approximation) ${ }^{1}$ :

\footnotetext{
${ }^{1}$ From time to time it is discussed in the literature, who was the first to introduce the quasiequilibrium approximations, and how to interpret them. At least a part of the discussion is due to a different role that quasiequilibrium plays in the entropy-conserving and dissipative dynamics. The very first use of the entropy maximization dates back to the classical work of Gibbs [1], but it was first claimed for a principle by Jaynes [2]. Probably the first explicit and systematic use of quasiequilibria to derive dissipation from entropy-conserving systems is due to the works of Zubarev. Recent detailed exposition is given in [3]. For dissipative systems, the use of the quasiequilibrium to reduce the description can be traced to the works of Grad on the Boltzmann equation [4]. The viewpoint of two of the present authors (ANG and IVK) was influenced by the papers by L.I. Rozonoer and co-workers, in particular, Refs. [5-7]. A detailed exposition of the quasiequilibrium approximation for Markov chains is given in the book [8] (Chapter 3, Quasiequilibrium and entropy maximum, pp. 92-122), and for the BBGKY hierarchy in the paper [9]. We have applied the maximum entropy principle to the description of the universal dependence of the 3-particle distribution function $F_{3}$ on the 2-particle distribution function $F_{2}$ in classical systems with pair interactions [10]. A very general discussion of the maximum entropy principle with applications to dissipative kinetics is given in the review [11].
} 
$S(\Psi) \rightarrow \max \quad M(\Psi)=M$,

where $S(\Psi)$ is the entropy. The quasiequilibrium closure is always thermodynamically consistent, but Problem 2 (the absence of the accuracy control) remains unsolved, and Problem 3 (the absence of refinement procedures) can be solved by adding new macroscopic variables to variational problem (2). But uncontrolled enlargement of the macroscopic variables set gives us no guarantee of the accuracy improvement. There exists one more specific problem for the quasiequilibrium approximation (2). Usually while solving variation problem (2) we can find explicit dependencies $\Psi(\Lambda)$ and $M(\Lambda)$, where $\Lambda$ are the corresponding Lagrange multipliers (dual variables), more or less easily. It is much more difficult to find the dependencies $\Lambda(M)$ and $\Psi(M)$ that we need for the closure of the macroscopic equations.

The method of Legendre integrators consists of building and solving the equations of motion for the dual variables. The methods of the first order, based on this idea, were suggested and tested in Refs. [12-14]. The method of the thermodynamic projector lets us represent every ansatz-manifold as the solution to the variational problem (2) with specially chosen constraints. The thermodynamic projector is the unique operator that transforms the arbitrary vector field equipped with the given Lyapunov function into a vector field with the same Lyapunov function. (This happens on any manifold that is not tangent to the level of the Lyapunov function.)

Equations that are derived by the method of the thermodynamic projector are always thermodynamically consistent. Although this idea was published in the year 1992 [16], the full construction is published only recently in application to chemical kinetics [17].

One of the problems, discussed in this paper, is to construct the method of the thermodynamic projector for the derivation of physically consistent macroscopic equations for the polymer dynamics. In the process of building the thermodynamic projector and the quasiequilibrium approximation one involves the Lyapunov function for the Eq. (1), which is the entropy $S$. The equations for the polymer dynamics (Fokker-Planck Equation) allows us to use the huge amount of different Lyapunov functions, and each of them can be formally chosen to describe the macroscopic processes. We need to analyze the different Lyapunov functions for the Fokker-Planck Equation.

The problem of accuracy estimation of the resulting approximations and their further improvement, it is suggested to solve with the procedures of post-processing. Suppose that for the dynamical system (1), the approximate invariant manifold has been constructed and the approximate slow motion equations $\Psi_{M}(t)$ have been derived:

$\frac{\mathrm{d} \Psi_{M}}{\mathrm{~d} t}=P_{\Psi_{M}}\left(J\left(\Psi_{M}\right)\right)$,

where $P_{\Psi_{M}}$ is the corresponding projector onto the tangent space $T_{\Psi_{M}}$ of $\Psi_{M}$. Suppose that we have solved system (3) and have obtained $\Psi_{M}(t)$. Let us consider the following two questions:

- How well does this solution approximate the true solution $\Psi(t)$ given the same initial conditions?

- How is it possible to use the solution $\Psi_{M}(t)$ for it's refinement without solving system (3) again?

These two questions are interconnected. The first question states the problem of accuracy estimation. The second one states the problem of post-processing. The corresponding methods to answer these questions are developed and described in this work.

\section{Elimination of fast variables with the help of the Lyapunov function}

The most popular way to investigate the dynamics of complicated systems is to split the motion into the slow and the fast components, and then to exclude the fast component. As a result, one gets a system of equations that describes the evolution of the slow variables. The necessary conditions of usefulness of this method are usually formulated as a set of restrictions for the possible dynamics of the "fast subsystem". Here the "fast subsystem" is the subsystem which describes the evolution of the fast variables with an assumption that slow variables are constant.

Unfortunately, there often appear situations where we cannot avoid using this method, and there is no proof that it is valid. These situations appear almost everywhere in physical kinetics. Here one follows the same scheme: the relaxation processes are split into slow and fast. In spite of the fact that in most cases the proofs of validity of this scheme are absent, the experience helps to avoid fatal errors.

In this section the method to obtain the equations of the macrokinetics from the micro-description is demonstrated. The basis of the analysis is the assumption that if the macroscopic variables are chosen in the proper way, then all other variables relax fast: the probability distribution of the microscopic variables after a small period of time is determined with good accuracy by the macroscopic variables. Let us call this assumption the "quasiequilibrium hypothesis".

The notion "macroscopic variables" is somewhat relative and is introduced to stress the difference of these variables from "everything else". For example, an one-particle distribution function can be "macroscopic" for the full description of the system.

The goal of this section is to describe the most primitive procedure of derivation of the equations for the slow variables and to discuss the form of these equations. In this paper, the reduction of description goes on with the help of the Lyapunov functions. This formalism is the case of the known principle of the conditional maximum of entropy with given values of the macroscopic variables.

Let us review the basic notions of the convex analysis, which are used here. The subset $U$ of the vector space $E$ is 
convex, if for every two points $x_{1}, x_{2} \in U$ it contains the segment between $x_{1}$ and $x_{2}$ : for every $\lambda \in[0,1]$

$\lambda x_{1}+(1-\lambda) x_{2} \in U$.

The intersection of any number of the convex sets is convex. The convex envelope of the subset $M$ of a vector space $E$ is the smallest convex set co $M \subset E$, that includes $M$. It is the intersection of all the convex sets, that include $M$.

If the set $U \subset E$ is convex and

$x_{1}, \ldots, x_{k} \in U, \lambda_{1}, \ldots, \lambda_{k} \geq 0, \sum_{i} \lambda_{i}=1$,

then $\sum_{i} \lambda_{i} x_{i} \in U$. this leads to another definition of the convex envelope:

$$
\begin{aligned}
\operatorname{co} M & =\left\{\sum_{i=1}^{k} \lambda_{i} x_{i} \mid x_{1}, \ldots, x_{k} \in M, \lambda_{1}, \ldots, \lambda_{k} \geq 0, \sum_{i} \lambda_{i}\right. \\
& =1, k<\infty\}
\end{aligned}
$$

If $\operatorname{dim} E=n$, then in Eq. (5) it is sufficient to take $k \leq n+1$ (Caratheodory Theorem).

The function $f$, defined on the convex set $U \subset E$, is convex, if its epigraph, i.e. the set of pairs

Epi $f=\{(x, g) \mid x \in U, g \geq f(x)\}$,

is the convex set in $E \times R$. Sometimes it is convenient to consider functions that can reach the value $f=\infty$. If there occurs a necessity to study the functions $f$ that are defined on the non-convex set $V \subset E$, then it is supposed that $f$ is convex, if the restriction of $f$ onto every convex subset of $V$ is convex. If the restriction of $f$ onto every line segment from the region of definition is convex, then $f$ is convex. The differentiable function $f$ of the class $C^{2}$ is convex if and only if the matrix of the second derivatives $\partial^{2} f / \partial x_{i} \partial x_{j}$ is nonnegative definite (i.e. all its eigenvalues are nonnegative). The smooth convex function $f$ on the convex set $U \subset R^{n}$ satisfies the inequality

$$
\begin{aligned}
& f\left(x^{1}\right)-f\left(x^{2}\right) \geq\left(\left.\nabla f\right|_{x^{2}}, x^{1}-x^{2}\right) \\
& \quad=\sum_{i}\left(\partial f / \partial x_{i}\right)_{x=x^{2}}\left(x_{i}^{1}-x_{i}^{2}\right),\left(x^{1}, x^{2} \in U\right)
\end{aligned}
$$

Geometrically this means that the graph of $f$ is located above the hyperplane, tangent at the point $x=x^{2}$.

The function $f$ is called strictly convex if in the domain of definition there is no line segment on which it is constant and finite $(f(x)=$ const $\neq \infty)$. The sufficient condition for the differentiable function $f$ of the $C^{2}$ class to be strictly convex is that the matrix of the second derivatives $\partial^{2} f / \partial x_{i} \partial x_{j}$ is positive defined.

In the set of the maximum points of the convex function $f$ on the compact set $U$ ( $U$ may be not convex) there are some boundary points of $U$, and if $U$ is convex, then there are some extreme points of $U$. The set of the minimum points of $f$ on the convex set $U$ is convex (but may be empty). The strictly convex continuous function has its maximum only in the boundary points of $U$, and if $U$ is convex, then in the extreme points. The strictly convex function may have a finite minimum only at one point. The function $f$ is called concave if the function $-f$ is convex. Every bounded convex function on the open subset of $R^{n}$ is continuous.

Let the $C^{2}$-smooth function $H$ be defined in the domain $U \subset R^{n}$. Let us relate the vector $\mu=\nabla_{x} H: \mu_{i}=\partial H / \partial x_{i}$ to every point $x \in U$. If the matrix $\partial \mu_{i} / \partial x_{j}=\partial^{2} H / \partial x_{i} \partial x_{j}$ is non-degenerated, then for the transform $x \rightarrow \mu$ there locally (in the neighborhood of every point) exists the differentiable inverse transform. The variables $\mu$ are often called conjugate variables, and the transform $x \rightarrow \mu$ is called "transition to the conjugated coordinates". Let the transform $x \rightarrow \mu$ be invertible on the open set $V \subset U$. This means that the function $x(\mu)$ is defined on $V$. Assuming the smoothness of this function, we describe the inverse transform $\mu \rightarrow x$ in the same way as the direct. For this purpose we introduce a function

$$
\begin{aligned}
& G(\mu)=(\mu, x(\mu))-H(x(\mu))=\sum_{i} \mu_{i} x_{i}(\mu)-H(x(\mu)) \\
& \frac{\partial G}{\partial \mu_{i}}=x_{i}+\sum_{j} \mu_{j} \frac{\partial x_{j}}{\partial \mu_{i}}-\sum_{j} \frac{\partial H}{\partial x_{j}} \frac{\partial x_{j}}{\partial \mu_{i}}=x_{i}
\end{aligned}
$$

The function $G$ is called the Legendre transform of $H$.

With the help of the conjugated coordinates it is possible to write down the necessary conditions of the extremum for problems with the linear constraints on the open set in a very simple way:

$H(x) \rightarrow \min , \quad \sum_{j} m_{i j} x_{j}=M_{i}, \quad(i=1, \ldots, k), x \in U(9)$

With the method of Lagrange multipliers we get the system of equations that gives us the necessary conditions for the solution to Problem (9):

$\mu_{j}=\sum_{i} \lambda_{i} m_{i j}, \quad j=1, \ldots, n$,
$\sum_{j} m_{i j} x_{j}=M_{i}, \quad(i=1, \ldots, k)$,

where the $\lambda_{i}$ are the Lagrange multipliers. The necessary conditions for the extremum are given by the system of Eq. (10). One part of the system is linear in the $x$ coordinates, and the other part is linear in the conjugated coordinates $\mu$.

Let us have the Legendre transform $G(\mu)$ for the function $H(x)$. Let the transform $x \rightarrow \mu$ have the smooth inverse transform, and let the solution to Problem (9) be unique for some open set of values of the vector $\left(M_{1}, \ldots, M_{k}\right) \in$ $R^{n}$. Also let the point of the minimum $x_{\min }$, and, consequently, the minimal value of $H$ be smoothly dependent on $M, H_{\min }=H(M)$. Let us denote $\mu_{M_{i}}=\partial H(M) / \partial M_{i}, \mu_{M}=$ $\left(\mu_{M_{1}}, \ldots, \mu_{M_{k}}\right)$. Let us get some information about the function $H(M)$ from the functions $H(x)$ and $G(\mu)$ without 
solving any equations. With the known value of the vector $\mu_{M}$ we can immediately find the vector $\mu$ at the corresponding point of the conditional minimum, $\mu_{j}=\sum_{i} \mu_{M i} m_{i j}$. From this equality we get

$x\left(\mu_{M}\right)=\left.\left(\nabla_{\mu} G(\mu)\right)\right|_{\mu_{j}=\sum_{i} \mu_{M_{i}} m_{i j}}$.

From $x_{\mu_{M}}$ we obtain $M\left(\mu_{M}\right)$ and $H\left(M\left(\mu_{M}\right)\right)$ :

$M_{i}\left(\mu_{M}\right)=\sum_{j} m_{i j} x_{j}\left(\mu_{M}\right), \quad H\left(M\left(\mu_{M}\right)\right)=H\left(x\left(\mu_{M}\right)\right)$.

Finally, the Legendre transform $G\left(\mu_{M}\right)$ for the function $H(M)$ is:

$G\left(\mu_{M}\right)=\left(\mu_{M}, M\left(\mu_{M}\right)\right)-H\left(M\left(\mu_{M}\right)\right)=G\left(\mu\left(\mu_{M}\right)\right)$.

So, we can find dependencies $\mu\left(\mu_{M}\right), x\left(\mu_{M}\right), M\left(\mu_{M}\right)$, $H\left(\mu_{M}\right)$ and $G\left(\mu_{M}\right)$ from the functions $H(x)$ and $G(x)$ without solving any equations. We hope that the similar notations for $H(x)$ and corresponding conditional minimum function $H(M)$, and for their Legendre transforms $G(\mu)$ and $G\left(\mu_{M}\right)$ will not cause confusion. Let us note that with our assumptions the reversibility of the transform $M \rightarrow \mu_{M}$ follows from the reversibility of the transform $x \rightarrow \mu$, and moreover, the function $M\left(\mu_{M}\right)$ can be found explicitly.

The convexity of the function $H(x)$ usually makes our assumptions (existence and uniqueness of the conditional minimum, global reversibility of the transform $x \rightarrow \mu$, smoothness of the function $H(M)$ ) easier to check. Note, that the convexity of the function $H(M)$ is neither a necessary nor a sufficient condition for our assumptions. If $H(x)$ is convex, then the function of the conditional minimum $H(M)$ is convex too.

Now we proceed to the problem of elimination of the fast variables. Let us have the system of differential equations with smooth right hand-sides.

$\dot{x}=F(x)$,

in the convex domain $U \subset R^{n}$, and moreover let the linear transform $x \rightarrow M, M_{i}=\sum_{j} m_{i j} x_{j}$ from the phase space to the space of the slow variables $M$ be defined. We can assume that we have no linearly dependent rows in the matrix $m_{i j}$, because it is always possible to eliminate the linearly dependent functions $M_{i}(x)$, if they are present.

Let us assume that in the interesting domain of initial conditions $x_{0}$ the solutions $x(t)$ of Eq. (14) are developing in the following way: the vector $x(t)$ is going rapidly to the value that is defined by the slow variables $M$; after that $x$ can be represented as a function of $M$ with good accuracy, and this function is unique for every initial condition. So,

(A) for each value of the slow variables $M \in M(U)$ there exist $x=x^{*}(M)$, such that if $M\left(x^{0}\right)=M^{0}$, then $x(t)$ is going very rapidly to some small neighborhood of the $x^{*}\left(M^{0}\right)$, and during that $M(x(t))$ is almost constant,

(B) in the process of the further evolution, $x(t)$ stays in the small neighborhood of the value of $x$ that corresponds to $M(x(t))$, so $x$ is close to $x^{*}(M(x(t)))$.

It is usually impossible to give a strong proof for (A) and (B) for situations of real complexity in nonequilibrium thermodynamics, so these assumptions are, probably, the weakest point of the entire construction. We are accepting them because we are sure that the evolution of the macroscopic variables can be described by the autonomous system of differential equations of the first order. (If it is impossible, then, probably, one should extend the list of macroscopic variables with respect to the physical properties of the investigated process.) There is another way to deal with this problem: to equip the approximations by the post-processing. The post-processing helps us to correct the errors, if they are not too big, and gives us a signal if they are too big.

If we know the function $x^{*}(M)$, then we can write

$\dot{M}=m F\left(x^{*}(M)\right), \quad \dot{M}_{i}=\sum_{j} m_{i j} F_{j}\left(x^{*}(M)\right)$.

In general, this equation can be used only for short periods of time which do not exceed some limit. The right-hand-side $m F\left(x^{*}(M)\right)$ of Eq. (15) is not exactly $m F(x(t))$, and it may cause an error increment; as a result the solution of the Eq. (15) will divert from the true solution strongly. The exclusion is the case when in accordance to Eq. (15) $M(t)$ tends to the only stable fixed point when $t \rightarrow \infty$. If the solution of Eq. (15) and the real values of $M(x(t))$ are not too far from one another during the time in which the solution of the Eq. (15) is approaching the small neighborhood of the fixed point, then Eq. (15) can be used also for $t \rightarrow \infty$.

The function $x^{*}(M)$ for the particular system is not unique, but the range of choices is small in the sense that the neighborhood of $x^{*}(M(x(t))$ ) (in which the evolution goes after the short period of time) is small.

Let us have the Lyapunov function $H(x)$ for the system (14) that is decreasing along the trajectories. We can try to find the dependence $x^{*}(M)$ as the solution to the problem $H(x) \rightarrow \min , m x=M$. This way seems to be natural, but it does not follow directly from assumptions A and B. For example, there could be a situation in which $H$ is very sensitive to small changes of the slow variables, and not sensitive to the changes of the fast variables. In this situation the assumption that $x^{*}(M)$ is the point of conditional minimum of the function $H$, may not give the desired result. The following idea does not solve the problem, but it can be useful: in applications, the system (14) usually depends on some parameters. It seems to be more reasonable to use the Lyapunov function that does not depend on these parameters, if there exists such a function. It is most important in the case when, among the parameters, we have such that their values are determinate, whether or not is it possible to split 
the variables into fast and slow. So, the fast variables will be eliminated with the help of the Lyapunov function.

Let us have the Lyapunov function $H$ for the initial system. Let the transform $x \rightarrow \mu=\nabla_{x} H$ have a smooth inverse, and let us know the Legendre transform $G(\mu)$ for the function $H(x)$. Here it is also assumed that for every $M \in M(U)$ Problem (9) has a unique solution, and the minimum point $x^{*}(M)$, and the function of the conditional minimum $H(M)$ smoothly depend on $M$. With the value $\mu_{M}=\nabla_{M} H(M)$ it is possible to find $\mu\left(\mu_{M}\right), x\left(\mu\left(\mu_{M}\right)\right)$ (see Eqs. (11) and (13)). The result is

$\dot{M}=\left.m F\left(\nabla_{\mu} G(\mu)\right)\right|_{\mu=\mu_{M} m}$,

where $\mu_{M} m$ is the product of the row vector $\mu_{M}$ and the matrix $m$ :

$\left(\mu_{M} m\right)_{j}=\sum_{i} \mu_{M_{i}} m_{i j}$

$\nabla_{\mu} G$ is the vector with components $\partial G / \partial \mu_{i}$, and all derivatives are taken at the point $\mu=\mu_{M} m$. The right-hand-sides of Eq. (16) are defined as the functions of $\mu_{M}$. In order to define them as functions of $M$, one needs to make the Legendre transform, find the function $H(M)$ and, respectively, $\mu_{M}=\nabla_{M} H(M)$ from the function $G\left(\mu_{M}\right)$ (13). It is impossible to make these calculations explicitly in such a general case. It seems to be very natural and convenient to define the right-hand-sides of the kinetic equations as functions of the conjugate variables. If in the beginning the right-hand-sides of Eq. (14) are defined as functions of $\mu$ (i.e. $\dot{x}=J(\mu)$ ), then Eq. (16) has a very simple form:

$\dot{M}=m J\left(\mu_{M} m\right)$.

$H(M)$ is the Lyapunov function for Eq. (16). Its time derivative due to the system (16) is not positive:

$\dot{H}(M)=\left(\mu_{M}, m J\left(\mu_{M} m\right)\right)=\left(\mu_{M} m, J\left(\mu_{M} m\right)\right) \leq 0$,

because $(\mu, J(\mu))=\dot{H}(x) \leq 0$.

Let us call the systems dissipative if $\dot{H} \leq 0$ and conservative, if $\dot{H}=0$. For the dissipative system we have $\dot{H}(M) \leq$ 0 (18), and if the system is conservative, then for all values of $\mu$ we have $(\mu, J(\mu))=\dot{H}(x)=0$. Then from Eq. (18) we get $\dot{H}(M)=\left(\mu_{M}, m J\left(\mu_{M} m\right)\right)=\left(\mu_{M} m, J\left(\mu_{M} m\right)\right)=0$. So, we proved the following theorem.

Theorem $^{2}$. The Lyapunov function for the microscopic system (14) remains the Lyapunov function for the macroscopic system (17), and if the microscopic system is conservative, then its quasiequilibrium projection to the space of macroscopic variables remains conservative.

\footnotetext{
2 This is a rather old theorem. One of us had published this theorem in 1984 already as textbook material ([8], Chapter 3 "Quasiequilibrium and entropy maximum", p. 37, see also the paper [14]), but from time to time different particular cases of this theorem continue to be published as new results.
}

If necessary, it is easy to perform further exclusion of the variables in Eq. (16) with the help of the function $H(M)$. The right-hand-sides of the resulting equations will be defined again as the functions of the conjugate variables, and the function of the conditional minimum will be the Lyapunov function again. Let us note that in Eq. (17) we have neither $H$ nor $G$ in explicit form. (They occur only when we need to find the connections between $M$ and $\mu_{M}$ or $x$ and $\mu$ ).

Convexity of $H$ was never used above, but the natural domain of applicability of the described formalism is composed of systems with convex Lyapunov functions $H(x)$, or at least with such $H$ that the sets $\{x \mid H(x)<h\}$ are convex. Otherwise there exist such linear manifolds, that the local minimum of $H$ is not unique on them, and further considerations are required to select the relevant minima. The finite dimensionality of phase space is not so important, because everything said above can be applied to the infinite-dimension case with proper restrictions. Let $E$ be the Banach space, $U \subset E$ be the convex open set, $H: U \rightarrow R$ be $C^{2}$-smooth function. With every point $x \in U$ we associate the linear functional $\mu_{x} \in E^{*}: \mu_{x}=\nabla_{x} H$, which is the differential of $H$ at the point $x$. Let $V$ be the set of values of $\mu_{x}$ for $x \in U$ and let us have the smooth mapping $J$ from $E^{*}$ to $E$ in the neighborhood of $V$. The system $(U, H, J)$ determines the system of equations

$\dot{x}=J\left(\mu_{x}\right)$

Let $L$ be the closed subset of $E$ and for every $M \in U / L$ let the problem $H(x) \rightarrow \min , x / L=M, x \in U$ have the unique solution $x_{\min }$, which is $C^{2}$-smooth dependent on $M$, $H(M)=H\left(x_{\min }\right)$. Denoting $\mu_{M}=\nabla_{M} H(M) \in(E / L)^{*} \in$ $E^{*}$ we can define the factor-system, which is the exact analogue of Eq. (16):

$\dot{M}=J\left(\mu_{M}\right) / L$

Here the argument $J$ is the linear functional on $\mu_{M}: \mu_{M} x=$ $\mu_{M}(x / L)$.

The described procedure of the elimination of variables has one very important commutativity property: if one makes a further simplification and transacts to the variables $N=$ $N(M)$, then after the application of the described formalism to the system (20) with the function $H(M)$, one gets the same result as after the application of this formalism directly to the reduction from $x$ to $N(x)=N(M(x))$. So, the chain of exclusions $x \rightarrow M \rightarrow N$ gives us the same result as the direct exclusion $x \rightarrow N$.

The Legendre transformation and maximization of thermodynamic Lyapunov functions (thermodynamic potentials) were recently placed by Grmela [15] at the physical basis of the nonequilibrium as well as of the equilibrium thermodynamic. Contact Hamiltonians give an elegant representation of the dynamics on Legendre manifolds [15]. 


\section{The main problems in usage of the quasiequilibrium approximations}

Our problem is to build the closed system

$\dot{M}=J(M)$,

from the initial system (1) and its Lyapunov function. If we know the function $x^{*}(M)$ then it is sufficient to calculate $m\left(F\left(x^{*}(M)\right)\right)$. This problem is the problem of calculation of the projection of the microscopic vector field $F$ on the macroscopic variables $M$ at known point $x^{*}(M)$. Let us call this problem the problem of macroscopic projection. If the right-hand-parts are expressed through $\mu$ then we have the problem of macroscopic projection too.

Another problem is to find $\mu_{M}$. Usually it is necessary to solve the system of non-linear equations (if the function $H$ is not quadratic) to solve this problem. Indeed, let us consider the conditions for the conditional extremum of $H$ with given values of the moments $M$. From the functions $H(x), G(\mu)$ we get $\mu\left(\mu_{M}\right), x\left(\mu_{M}\right), M\left(\mu_{M}\right), H\left(M\left(\mu_{M}\right)\right)$. But in this list we have no function $\mu_{M}(M)$. We can find this function as the solution of the equation

$M\left(\mu_{M} m\right)=M$

Let us give a few examples.

One-particle approximation: Let $x$ be the $N$-particle distribution function, $f_{N}\left(\xi_{1}, \ldots, \xi_{N}\right)$, where $\xi_{i}$ is vector of coordinates and momenta of the $i$ th particle, and let the evolution of this function be described by the linear equation

$\frac{\partial f_{N}}{\partial t}=L f_{N}$.

Furthermore, let $M$ be the one-particle distribution function

$f_{1}(\xi)=N \int f_{N}\left(\xi, \xi_{2}, \ldots, \xi_{N}\right) \mathrm{d} \xi_{2}, \ldots, \mathrm{d} \xi_{N}$,

and $H$ be the entropy (we use the $H$-function which is equal to negative entropy)

$H\left(f_{N}\right)=\int f_{N}\left(\ln f_{N}-1\right) \mathrm{d}^{N} \xi$

For given $f_{N}, H, f_{1}$, we get $\mu=\ln f_{N}, f_{N}=\exp \mu$,

$G(\mu)=\int \exp \mu\left(\xi_{1}, \ldots, \xi_{N}\right) \mathrm{d}^{N} \xi$,

$m\left(f_{N}\right)=\int \sum_{i=1}^{N} \delta\left(\xi-\xi_{i}\right) f_{N}\left(\xi_{1}, \ldots, \xi_{N}\right) \mathrm{d}^{N} \xi ;$ the extremum conditions (10) are of the form

$\mu\left(\xi_{1}, \ldots, \xi_{N}\right)=\int \mathrm{d} \xi \mu_{1}(\xi) \sum_{i=1}^{N} \delta\left(\xi-\xi_{i}\right)=\sum_{i} \mu_{1}\left(\xi_{i}\right)$,

$f_{N}=\exp \sum_{i} \mu_{1}\left(\xi_{i}\right)$

The normalization condition here is $\int f_{N} \mathrm{~d}^{N} \xi=1$, that is

$\int \exp \mu_{1}(\xi) \mathrm{d} \xi=1$
Connection between the macroscopic variables $f_{1}$ (that is $M)$ and the quasiequilibrium values of the microscopic variables $f_{N}^{*}$ (that is, $x_{M}^{*}$ ) is given by the well-known formula:

$f_{N}\left(\xi_{1}, \ldots, \xi_{N}\right)=\frac{1}{N^{N}} f_{1}\left(\xi_{1}\right), \ldots, f_{1}\left(\xi_{N}\right)$

Projection of the microscopic vector field (22) can be found by direct integration.

Two-particle distribution function as the macroscopic variable: The one-particle distribution function $f_{1}(\xi)$ is often not sufficient because, for example, the energy of interaction of pairs of particles cannot be found from this function. A much more detailed description is given by the two-particle distribution function,

$f_{2}\left(\xi_{1}, \xi_{2}\right)=N(N-1) \int f_{N}\left(\xi_{1}, \ldots, \xi_{N}\right) \mathrm{d} \xi_{3}, \ldots, \mathrm{d} \xi N$

We can easily find the expression

$\mu\left(\xi_{1}, \ldots, \xi_{N}\right)=\sum_{i, j, i \neq j} \mu_{2}\left(\xi_{i}, \xi_{j}\right)$,

$f_{N}\left(\xi_{1}, \ldots, \xi_{N}\right)=\exp \mu=\exp \sum_{i, j, i \neq j} \mu_{2}\left(\xi_{i}, \xi_{j}\right)$,

but it is difficult to find the connection between $\mu_{2}$ and $f_{2}$ explicitly. Only a series expansion in the neighborhood of the uncorrelated state is known [10]. The problem with the macroscopic projection becomes hard too: the necessary integrals in the general case are impossible to find analytically. For two-particle distribution functions as well as for the majority of the most interesting variables the transform $M \leftrightarrow \mu_{M}$ is very complicated in the forward direction and not very simple (as simple as the derivation of $f_{2}$ from $f_{N}$ ) in the opposite direction. So, we need to avoid the necessity of calculating $\mu_{M}(M)$ (and, if possible, to make less calculations to find $M\left(\mu_{M}\right)$ ).

The first of these two problems (avoiding calculation of $\mu_{M}(M)$ ) is solved by the method of Legendre integrators which is developed in Ref. [12].

\section{Legendre integrators}

The main idea of Legendre integrators is to find some alternate way to solve the macroscopic equations $\dot{M}=J(x)$ : a way to find their solution in the absence of the explicit form of these equations. First of all, note, that we have a linear connection between $\dot{M}$ and $\dot{\mu}_{M}$ :

$\frac{\mathrm{d} M}{\mathrm{~d} t}=\left(m\left(D_{x}^{2} S(x)\right)^{-1} m^{\mathrm{T}}\right) \frac{\mathrm{d} \mu_{M}}{\mathrm{~d} t} ;$

$\dot{M}=\frac{\mathrm{d}}{\mathrm{d} t}\left(m x\left(\mu_{M} m\right)\right)=m\left(D_{\mu} x\right) m^{\mathrm{T}} \dot{\mu} ;$

$D_{\mu} x=\left(D_{x} \mu\right)^{-1}=\left(D_{x}^{2} S(x)\right)^{-1}$ 
Calculation of the functions $m(F(x))$ is the standard problem of the macroscopic projection. Dependencies $x\left(\mu_{M}\right)$ are usually quite simple. We suggest the following chain of computations to solve the (unknown) equations $\dot{M}=\Phi(M)$ :

$$
\begin{aligned}
\mu_{M}(t) & \rightarrow x=x\left(\mu_{M}\right) \rightarrow \dot{M} \rightarrow \dot{\mu}_{M} \\
& \rightarrow \mu_{M}(t+\Delta t) \rightarrow M(t+\Delta t)
\end{aligned}
$$

In the sequence (32) there is one operation of macroscopic projection and one operation of solving the system of linear Eq. (30).

Formally, it is possible to write down the equations for $\mu_{M}$ :

$\frac{\mathrm{d} \mu_{M}}{\mathrm{~d} t}=\left(m\left(D_{x}^{2} S(x)\right)^{-1} m^{\mathrm{T}}\right)^{-1} m F(x)$,

where $x=x_{M}^{*}$.

Nevertheless, explicit inversion of the operator in the right-hand-part of Eq. (33) is usually difficult and one should use the chain of computations (32). In our first calculations using Legendre integrators $[12,13]$ the methods of first-order of accuracy were used. This is not the principal restriction: the scheme (32) gives us a possibility to calculate $\dot{\mu}_{M}$ for any given $\mu_{M}$, so all known methods of higher-order can be used (for example, the Runge-Kutta method with different procedures of automatic step selection [25-27]).

\section{Lyapunov functions for the Fokker-Planck equation}

The Fokker-Planck equation (FPE) in the absence of the driving forces has the form

$\frac{\partial \Psi(q, t)}{\partial t}=\nabla_{q}\left\{D\left(\Psi(q, t) \nabla_{q} U(q)+\nabla_{q} \Psi(q, t)\right)\right\}$,

where $\Psi$ is the probability density over the configuration space, $q$ is a point in this space, $\Psi(q)$ is the function of time $t, U(q)$ is the normalized potential energy $(U=$ $\left.U_{\text {potential }} / k T\right), D(q)$ is the positively semidefinite diffusion operator $\left(\left(y, D_{y}\right) \geq 0\right)$.

The FPE has two important properties:

(1) Conservation of the total probability:

$$
\frac{\mathrm{d}}{\mathrm{d} t} \int \Psi(q, t) \mathrm{d} q \equiv 0
$$

(2) Dissipation: for every convex function of one variable $h(a)\left(h^{\prime \prime}(a)>0, a \geq 0\right)$ the following functional $S[\Psi]$ is monotonically non-increasing in time:

$$
S[\Psi]=-\int \Psi^{*}(q) h\left(\frac{\Psi(q)}{\Psi^{*}(q)}\right) \mathrm{d} q,
$$

where

$\Psi^{*}(q)=$ const $\cdot \exp (-U(q))$,

is the Boltzmann-Gibbs distribution.
For $h(a)=a \ln a$, the functional $S[\Psi]$ is the usual Boltzmann-Gibbs-Shannon entropy:

$S[\Psi]=-\int \Psi^{*}(q) \ln \left(\frac{\Psi(q)}{\Psi^{*}(q)}\right) \mathrm{d} q$

Let us calculate the time derivative of $S[\Psi]$ due to FPE (34). Note, that

$\nabla_{q}\left(\frac{\Psi(q)}{\Psi^{*}(q)}\right)=\frac{\nabla_{q} \Psi(q)+\Psi(q) \nabla_{q} U}{\Psi^{*}(q)}$,

so we can rewrite FPE as follows:

$\frac{\partial \Psi(q, t)}{\partial t}=\nabla_{q} D\left(\Psi^{*}(q) \nabla_{q}\left(\frac{\Psi(q)}{\Psi^{*}(q)}\right)\right)$

Let us consider FPE in the domain $\Omega$. Function $\mathrm{d} S / \mathrm{d} t$ consists of two summands: the first is the integral of the local "production of $S$ ", $\int \sigma(q) \mathrm{d} q$, and the second is the flow through the boundary of the domain $\Omega$ :

$$
\begin{aligned}
\frac{\mathrm{d} S(\Psi)}{\mathrm{d} t}= & -\int_{\Omega} h^{\prime}\left(\frac{\Psi}{\Psi^{*}}\right) \nabla_{q}\left(D \Psi^{*}\left(\nabla_{q}\left(\frac{\Psi}{\Psi^{*}}\right)\right)\right) \mathrm{d} q \\
= & -\int_{\Omega} \operatorname{div}\left[h^{\prime}\left(\frac{\Psi}{\Psi^{*}}\right) D \Psi^{*} \nabla_{q}\left(\frac{\Psi}{\Psi^{*}}\right)\right] \mathrm{d} q \\
& +\int_{\Omega} \Psi^{*} h^{\prime \prime}\left(\frac{\Psi}{\Psi^{*}}\right)\left(\nabla_{q}\left(\frac{\Psi}{\Psi^{*}}\right), D \nabla_{q}\left(\frac{\Psi}{\Psi^{*}}\right)\right) \mathrm{d} q \\
= & \int_{\partial \Omega} \Psi^{*} h^{\prime}\left(\frac{\Psi}{\Psi^{*}}\right)\left(v_{q}, D \nabla_{q}\left(\frac{\Psi}{\Psi^{*}}\right)\right) \mathrm{d} w \\
& +\int_{\Omega} \sigma(q) \mathrm{d} q,
\end{aligned}
$$

where $d w$ is the differential of the area, $v_{q}$ is a vector of the unitary normal to $\partial \Omega$ in the point $q, \sigma(q)$ is the entropy $S$ production:

$\sigma(q)=\Psi^{*} h^{\prime \prime}\left(\frac{\Psi}{\Psi^{*}}\right)\left(\frac{\Psi}{\Psi^{*}}, D \nabla_{q}\left(\frac{\Psi}{\Psi^{*}}\right)\right) \geq 0$

Let the flow of $\Psi$ through the boundary $\partial \Omega$ be equal to zero:

$\left(v_{q}, D \nabla_{q}\left(\frac{\Psi}{\Psi^{*}}\right)\right)=0$,

at all points of $\partial \Omega$. Then

$\frac{\mathrm{d} S}{\mathrm{~d} t}=\int_{\Omega} \sigma(q) \mathrm{d} q \geq 0$

The most important cases of $S$ selection are: $h(a)=a \ln a$, $S$ is the Boltzmann-Shannon-Gibbs entropy; $h(a)=$ $\beta a \ln a-(1-\beta) \ln a, 0 \leq \beta \leq 1$ is the maximal family of additive trace-form entropies [18-20] (these entropies are additive for composition of independent subsystems); $h(a)=\left(1-a^{\beta}\right) /(1-\beta), \beta \neq 1$ is the Tsallis entropy [21]. These entropies are not additive, but become additive after nonlinear monotonic transformation. This property can serve as a definition of the Tsallis entropies in the class of generalized entropies (36) [20]. 


\section{Macroscopic variables and quasiequilibrium distribution functions for the FPE}

The set of the macroscopic variables can be continuous or discrete. Let $\alpha$ be the discrete or continuous parameter that enumerates the macroscopic variables, and $M_{\alpha}$ be the corresponding variables. Every macroscopic value $M_{\alpha}$ is defined by its microscopic density $m_{\alpha}(q)$ :

$M_{\alpha}=\int_{\Omega} m_{\alpha}(q) \Psi(q) \mathrm{d} q$

The choice of the domain $\Omega$, in which we are solving the FPE, needs to be discussed separately. We can suppose formally, that $\Omega=R^{n}$, but for the calculations it is better to make it as small as possible for the preservation of accuracy. Usually, when $\|q\| \rightarrow \infty$ the function $\Psi(q)$ tends to zero faster than exponentially, and we can a priori select the bounded domain $\Omega$, out of which $\Psi$ is negligibly small. We shall do the calculations for the general form of $S$ (see Eq. (36)) and give the examples for the most popular choice (38) of $S$.

The quasiequilibrium function $M_{\alpha}$ for the given Lyapunov function $S$ (36) is defined as the solution to the problem

$S(\Psi) \rightarrow \max , \quad \int m_{\alpha}(q) \Psi(q) \mathrm{d} q=M_{\alpha}$

Due to the convexity of $h$ (and, consequently, concavity of $S$ ), it is sufficient to investigate the conditions of the local extremum:

$D_{\Psi} S=\sum_{\alpha} m_{\alpha}(q) \mu_{\alpha}$

where $\mu_{\alpha}$ are variables that are dual to $M_{\alpha}\left(\mu_{M}\right)$. For a continuous parameter the sum in Eq. (42) is replaced by integration on $\alpha$.

Next, we use the standard Riesz representation of functionals (through the $L^{2}$ scalar product). Let us write

$D_{\Psi} S(\Psi)=-h^{\prime}\left(\frac{\Psi}{\Psi^{*}}\right) ; \quad h^{\prime}\left(\frac{\Psi}{\Psi^{*}}\right)=-\sum_{\alpha} m_{\alpha}(q) \mu_{\alpha}$

For the quasiequilibrium distribution we have

$\Psi=\Psi^{*} g\left(-\sum_{\alpha} m_{\alpha}(q) \mu_{\alpha}\right)$,

where $g(a)$ is a function of one variable, inverse to $h^{\prime}(b)$. Note that $h^{\prime}(b)$ is a monotonically increasing function (because $h$ is convex), so $g(a)$ is a monotonically increasing function too, and $g^{\prime}(a)=\left(h^{\prime \prime}(g(a))\right)^{-1}$. Let us denote the quasiequilibrium distribution function (43) as $\Psi^{\mathrm{qe}}\left(\left\{\mu_{\alpha}\right\}, q\right)$.

For the BGS entropy $h(b)=b(\ln b-1), h^{\prime}(b)=\ln b$, $g(a)=\exp a$, and Eq. (43) transforms into the following equation:

$\Psi^{\mathrm{qe}}\left(\mu_{\alpha}, q\right)=\Psi^{*} \exp \left(-\sum_{\alpha} m_{\alpha}(q) \mu_{\alpha}\right)$
For the next steps it is convenient to consider the temperature dependence explicitly (i.e. write $\beta U$ instead of $U$ in FPE, $\beta=1 / k T)$, then we have $\Psi^{*}=$ const $\cdot \exp (-\beta U)$.

For the classical BGS entropy (38) the quasiequilibrium distribution will take the simplest form

$\Psi^{\mathrm{qe}}\left(\left\{\mu_{\alpha}\right\}, q\right)=\exp \left(-\mu_{0}-\mu_{U} U-\sum_{\alpha} m_{\alpha}(q) \mu_{\alpha}\right)$,

where $\mu_{U}=\beta=1 / k T, \mu_{0}$ is a variable, conjugated to $M_{0}=\int_{\Omega} \Psi \mathrm{d} q \equiv 1$. The function (45) is a solution to the problem

$$
\begin{aligned}
& -\int_{\Omega} \Psi \ln \Psi \mathrm{d} q \rightarrow \max , M_{0}(\Psi)=\int_{\Omega} \Psi(q) \mathrm{d} q \\
& \quad=1, M_{U}(\Psi)=\int_{\Omega} U(q) \Psi(q) \mathrm{d} q=M_{U}, M_{\alpha}(\Psi) \\
& \quad=\int_{\Omega} m_{\alpha}(q) \Psi(q) \mathrm{d} q=M_{\alpha}
\end{aligned}
$$

In Eq. (46) we move from the relative (so-called Kullback) entropy to the absolute entropy.

Selection of the macroscopic variables is the most critical point in construction of quasiequilibrium approximations. It is always necessary to select them based on the specific problem. Nevertheless, there are some simple general recommendations about construction of the set of variables for the Legendre integrators.

(1) It is necessary to include $M_{0}$ in the list of variables, because $\mu_{0}$ is not constant in time.

(2) It is useful to include $M_{U}$ in the list of variables. With this variable in the process of the relaxation, all other $\mu_{\alpha} \rightarrow 0$ and $\mu_{U} \rightarrow 1 / k T$.

(3) It is better for the set of functions $m_{\alpha}(q)$ to be linearly independent.

For the classical entropy we have

$$
\begin{aligned}
& \Psi^{\mathrm{qe}}(\mu, q, t) \\
& \quad=\exp \left(-\mu_{0}(t)-\mu_{U}(t) U(q)-\sum_{\alpha} m_{\alpha}(q) \mu_{\alpha}(t)\right)
\end{aligned}
$$

Due to Eq. (47) we have

$$
\frac{\partial \Psi}{\partial t}=-\Psi\left[\frac{\mathrm{d} \mu_{0}}{\mathrm{~d} t}+U(q) \frac{\mathrm{d} M_{U}}{\mathrm{~d} t}+\sum_{\alpha} m_{\alpha}(q) \frac{\mathrm{d} \mu_{\alpha}}{\mathrm{d} t}\right]
$$

The FPE gives us

$$
\begin{aligned}
\frac{\partial \Psi}{\partial t}= & \nabla D\left(\Psi^{*} \nabla \frac{\Psi}{\Psi^{*}}\right)=-\Psi\left[\left(\mu_{U}-\beta\right)(\nabla, D \nabla) U(q)\right. \\
& +\sum_{\alpha} \mu_{\alpha}(\nabla, D \nabla) m_{\alpha}(q) \\
& -\sum_{\alpha}\left(2 \mu_{U} \mu_{\alpha}-\beta \mu_{\alpha}\right)\left(\nabla U(q), D \nabla m_{\alpha}(q)\right) \\
& \left.-\sum_{\alpha, \alpha^{\prime}} \mu_{\alpha} \mu_{\alpha^{\prime}}\left(\nabla m_{\alpha}(q), D \nabla m_{\alpha^{\prime}}(q)\right)\right]
\end{aligned}
$$


To calculate $\mathrm{d} M / \mathrm{d} t(\{\mu\})$ one has to calculate the following integrals:

$$
\frac{\mathrm{d} M_{U}}{\mathrm{~d} t}=\int_{\Omega} U(q) \frac{\partial \Psi(q)}{\partial t} \mathrm{~d} q ; \quad \frac{\mathrm{d} M_{\alpha}}{\mathrm{d} t} \int_{\Omega} m_{\alpha}(q) \frac{\partial \Psi(q)}{\partial t} \mathrm{~d} q,
$$

where $\partial \Psi / \partial t$ is calculated with Eq. (49), $\mathrm{d} M_{0} / \mathrm{d} t=0$.

From Eq. (48) we get the conditions for derivation of $\dot{\mu}$

$$
\begin{aligned}
& -\frac{\mathrm{d} \mu_{0}}{\mathrm{~d} t}-M_{U} \frac{\mathrm{d} \mu_{U}}{\mathrm{~d} t}-\sum_{\alpha} M_{\alpha} \frac{\mathrm{d} \mu_{\alpha}}{\mathrm{d} t}=\dot{M}_{0}=0 \\
& -M_{U} \frac{\mathrm{d} \mu_{0}}{\mathrm{~d} t}-\left\langle U^{2}\right\rangle_{\Psi} \frac{\mathrm{d} \mu_{U}}{\mathrm{~d} t}-\sum_{\alpha}\left\langle U m_{\alpha}\right\rangle_{\Psi}=\dot{M}_{U} ; \\
& -M_{\alpha} \frac{\mathrm{d} \mu_{0}}{\mathrm{~d} t}-\left\langle U m_{\alpha}\right\rangle_{\Psi} \frac{\mathrm{d} \mu_{U}}{\mathrm{~d} t}-\sum_{\gamma}\left\langle m_{\gamma} m_{\alpha}\right\rangle_{\Psi} \frac{\mathrm{d} \mu_{\gamma}}{\mathrm{d} t}=\dot{M}_{\alpha},
\end{aligned}
$$

where by $\langle f(q) g(q)\rangle_{\Psi}$ we denote the averaging $\langle f g\rangle_{\Psi}=$ $\int_{\Omega} f(q) g(q) \Psi(q) \mathrm{d} q$. We get the closed system for derivation of the dynamics of $\mu$. But the question about the choice of the macroscopic variables still remains open.

In the problem of quasiequilibrium we find the projections of $\Psi$ to the given set of the functions (linear space); afterwards we calculate $\Psi$ due to the maximum entropy condition. It seems to be physically sensible to choose the additional variables to $M_{0}, M_{U}$ as the projections of $\Psi$ onto some equilibrium states:

$M_{\alpha}(\Psi)=\int_{\Omega} \mathrm{e}^{-\alpha U(q)} \Psi(q) \mathrm{d} q$

There are two classical choices of macroscopic variables:

(1) $\alpha=R_{+}$(Laplace transform of the energy distribution density).

(2) $\alpha=i k, k \in R$ (Fourier transform of the energy distribution density).

The variable $M_{U}$ is the average energy in the potential well $U(q)$. In analogy to this, the variable $M_{\alpha}(\Psi)(51)$ for the real $\alpha>0$ can be considered as the energy in the potential well $\mathrm{e}^{-\alpha U(q)}$. This potential is gained by the monotonic nonlinear deformation of the energy scale $U \rightarrow \mathrm{e}^{-\alpha U(q)}$. For imaginary $\alpha$ this nonlinear deformation is given by the periodical functions $U \rightarrow \cos (k U)+i \sin (k U)$.

A benefit of using Eq. (51) is also in that $\left\langle m_{\alpha} m_{\alpha^{\prime}}\right\rangle=$ $M_{\alpha+\alpha^{\prime}}$, and we have to perform less calculations in (50). This set of the deformed energies can be used for both the initial potential $U$ and the set of additional potentials.

Is this set of macroscopic variables sufficient for the description of nonequilibrium kinetics of polymers in the presence of flow? Probability densities for all the quasiequilibrium distributions that can be constructed with these macroscopic variables have the form $\Psi(q)=\varphi(U(q))$, where $\varphi(U)$ is a function of one variable. Is this class of distributions sufficient for the specific problem? This question can be answered only after specification of the problem. But what is it possible to do, if the closure with these variables gives too large of an error (the estimation of accuracy is discussed below)? There are at least two ways: to extend the list of variables or to improve the quasiequilibrium manifold [14,34]. (Application of the methods of invariant manifolds to improving the quasiequlibrium closure for dynamics of dilute polymeric solution is presented in [35].) The extension of the list of variables is the central method of extended irreversible thermodynamics [36]. It is possible to combine the potential energy $U(q)$, the vector of the configuration space $q$, and the gradient of $U(q), \nabla U(q)=-F(q),(F(q)$ is the force) to obtain a huge amount of densities $m(q)$, which can be scalars, vectors, or tensors. The corresponding "macroscopic variables" are $\int_{\Omega} m(q) \Psi(q) \mathrm{d} q$.

The best hint for a choice of new macroscopic variables is the analysis of the right-hand-side of the dynamic equations [37]. The well-known distinguished macroscopic variable associated with the polymeric kinetic equations is the polymeric stress tensor $[33,40]$. This variable is not the conserved quantity but nevertheless it should be treated as a relevant slow variable because it actually contributes to the macroscopic (hydrodynamic) equations. Equations for the stress tensor are known as "constitutive equations", and the problem of reduced description for the polymeric models consists in deriving such equations from the kinetic equation.

The tensor

$\tau_{\mathrm{p} i j}=k_{\mathrm{B}} T\left(\delta_{i j}-\int_{\Omega} F_{i} q_{j} \Psi(q) \mathrm{d} q\right)$

gives a contribution to stresses caused by the presence of polymer molecules for unit density. Here $F(q)=-\nabla U(q)$ is the force vector, $\delta_{i j}$ is the Kronecker symbol. For spherically symmetric potentials $\left(U(q)=u\left(q^{2}\right)\right)$ this tensor is symmetric. The tensor of densities $m_{i j}(q)=F_{i}(q) q_{j}$ is the first addition to the densities that depend only of $U(q)$.

For the Boltzmann equation the thorough study of different types of the macroscopic description based on the analysis of the right-hand-part of the equation was provided in Refs.[38,39]. The first type involves only moments of distribution functions. It is the strategy used in the extended irreversible thermodynamics [36]. The second type of description involves only collision moments [38,39]. Finally, the third type involves both the moments and the collision moments (the mixed description). The second and the mixed hydrodynamics are sensitive to the choice of the collision model. It is shown, in particular, that the complete account of scattering processes leads to a renormalization of transport coefficients. Explicit method of constructing of approximations is developed for strongly nonequilibrium problems. This method enables one to treat any complicated nonlinear functionals that fit into the physics of a problem (such as, for example, rates of processes) as new independent variables. 


\section{Macroscopic variables and boundary conditions}

There is a standard technique to solve boundary value and initial-boundary value problems of mathematical physics: first, build the space of the functions that satisfy the boundary conditions, and then find the solution in this space. When one uses the Legendre integrators, a special technique is needed to satisfy the boundary conditions.

The FPE describes the evolution of the probability distribution. It conserves the total probability. The natural boundary conditions for the FPE is the absence of flow through the boundary of $\Omega$ :

$\Psi^{\mathrm{qe}}\left(v_{q}, D \nabla_{q}\left(\frac{\Psi}{\Psi_{q}}\right)\right)=0$

on $\partial \Omega$, where $v_{q}$ is a vector of outlet normal to $\partial \Omega$ in the point $q$. Quasiequilibrium distribution functions (47) satisfy the condition (53), if

$\left(v_{q}, D \nabla_{q} U(q)\right)=0,\left(v_{q}, D \nabla_{q} m_{\alpha}(q)\right)=0$,

for all $\alpha$.

There is also a different way to satisfy conditions (53): to make $\left.\Psi^{*}\right|_{\partial \Omega}=0$. It is possible to do so by making $U(q) \rightarrow$ $\infty$ while $q \rightarrow q_{0} \in \partial \Omega$. But this choice leads to singularities and is very inconvenient from the numerical point of view.

Conditions (54) look somewhat surprisingly, if considered outside of the context of quasiequilibrium approximations: for quasiequilibrium solutions the absence of flow through the barrier follows not from the infinite heights of the barrier, but from the fact, that the normal derivatives of $U$ and $m_{\alpha}$ are zeros.

To satisfy the condition (54) it may be necessary to deform the initial potential $U$ and densities $m(q)$. This deformation will be smoothing of $U$ near $\partial \Omega$. The error introduced by this deformation is usually not very big (because of the smallness of $\Psi^{*}$ near $\partial \Omega$ ) and can be estimated easily.

So, the quasiequilibrium approximation and the Legendre integrators of any order of accuracy are built, and the way to satisfy the boundary conditions is suggested. Numerical experiments $[12,13]$ have proven the effectiveness of this idea. The main computational challenge in this method is to calculate integrals of the form

$\int_{\Omega}\left(\sum \lambda_{k} \varphi_{k}(q)\right) \exp \left(\sum \gamma_{i} \Psi_{i}(q)\right) \mathrm{d} q$

where $\varphi_{k}(q), \Psi_{i}(q)$ are known functions. (Usually they are given analytically.) For problems of polymer physics the complexity of the problem (55) is dependent on two characteristics:

(1) The number of the different functions $\varphi_{k}(q), \Psi_{i}(q)$ is usually $5-10$.

(2) The dimension of the space in which the integration is performed is usually $10-100$.

\section{Thermodynamic projector and Galerkin approximations}

Almost every manifold of functions can be represented as the solution to the quasiequilibrium problem (41), if this manifold is not tangent to the level surface of the entropy $S=$ const [16]. For this representation only the right system of restrictions is needed. By simple parameterization with the moments $M(\Psi)$ it is possible to get only the classical quasiequilibrium manifolds (41). The restrictions that are necessary to represent manifold $\Omega$ as the quasiequilibrium manifold are built as follows. Let $f \in \Omega$, and $T_{f}$ be the tangent space to $\Omega$ in the point $f$. On the space of the distribution functions $E$ we define the projector $P_{f}: E \rightarrow$ $T_{f}$. Operator $P_{f}$ depends smoothly on the point $f$ and on $T_{f}$. The problem of quasiequilibrium is posed as follows:

$S(\Psi) \rightarrow \max , P_{f}(\Psi-f)=0$.

The necessary and sufficient condition for $f$ to be the unique solution to the problem (56)is [16]:

$\left.\operatorname{ker} P_{f} \subseteq \operatorname{ker} D_{\Psi} S\right|_{f}$,

that is, if $P_{f}(\varphi)=0$, then $\left.D_{\Psi} S\right|_{f}(\varphi)=0$. For the classical entropy

$\left.D_{\Psi} S\right|_{f}(\varphi)=-\int \varphi(q) \ln f(q) \mathrm{d} q$

and the condition (57) takes the form:

if $P_{f}(\varphi)=0$, then $\int \varphi \ln f \mathrm{~d} q=0$.

Among all projectors that satisfy the condition (57) there is unique projector, which has the following property. Let us have the appropriate equation

$\dot{\Psi}=J(\Psi)$,

for which $\mathrm{d} S[\Psi] / \mathrm{d} t \geq 0$. Then for the projected equation on $\Omega$

$\dot{f}=P_{f}(J(f))$,

we also have $\mathrm{d} S[f] / \mathrm{d} t \geq 0$. This projector was introduced in the paper [17], and there its uniqueness was also demonstrated. It is built as follows.

Let us require that the field of projectors, $P(\Psi, T)$, is defined for any $\Psi$ and $T$, if

$T \not \subset \operatorname{ker} D_{\Psi} S$.

From these conditions it follows immediately that at equilibrium, $P\left(\Psi^{*}, T\right)$ is the orthogonal projector onto $T$ (orthogonality with respect to entropic scalar product $\langle\mid\rangle_{\Psi^{*}}$ ).

The field of projectors is constructed in the neighborhood of equilibrium based on the requirement of maximal smoothness of $P$ as a function of $g_{\Psi}=D_{\Psi} S$ and $\Psi$. It turns out that to the first order in the deviations $\Psi-\Psi^{*}$ and $g_{\Psi}-g_{\Psi^{*}}$, the projector is defined uniquely. Let us first describe the construction of the projector, and next discuss its uniqueness. 
Let the subspace $T \subset E$, the point $\Psi$, and the differential of the entropy at this point, $g_{\Psi}=D_{\Psi} S$, be defined such that the transversality condition (61) is satisfied. Let us define $T_{0}=T \bigcap \operatorname{ker} g_{\Psi}$. By the condition (61), $T_{0} \neq T$. Let us denote $e_{g}=e_{g}(T) \in T$ as the vector in $T$, such that $e_{g}$ is orthogonal to $T_{0}$, and is normalized by the condition $g\left(e_{g}\right)=$ 1. Vector $e_{g}$ is defined unambiguously. Projector $P_{S, \Psi}=$ $P(\Psi, T)$ is defined as follows: for any $z \in E$,

$P_{S, \Psi}(f)=P_{0}(z)+e_{g} g_{\Psi}(f)$,

where $P_{0}$ is the orthogonal projector on $T_{0}$ (orthogonality with respect to the entropic scalar product $\left.\langle\mid\rangle_{\Psi}\right)$. Entropic projector (62) depends on the point $\Psi$ through the $\Psi$-dependence of the scalar product $\langle\mid\rangle_{\Psi}$, and also through the differential of $S$ in $\Psi$, the functional $g_{\Psi}$.

Obviously, $P(f)=0$ implies $g(f)=0$, that is, the thermodynamic requirement is satisfied. Uniqueness of the thermodynamic projector (62) is supported by the requirement of the maximal smoothness [17] of the projector as a function of $g_{\Psi}$ and $\langle\mid\rangle_{\Psi}$, and is done in two steps, which we sketch here:

(1) Considering the expansion of entropy at equilibrium up to quadratic terms, one shows that at equilibrium the thermodynamic projector is the orthogonal projector with respect to the scalar product $\langle\mid\rangle_{\Psi^{*}}$.

(2) For a given $g$, one considers auxiliary dissipative dynamic systems that satisfy the condition: for every $\Psi^{\prime} \in$ $U$, it holds $g_{\Psi}\left(J\left(\Psi^{\prime}\right)\right)=0$; that is, $g_{\Psi}$ defines an additional linear conservation law for the auxiliary systems. For the auxiliary systems, the point $\Psi$ is the equilibrium. Eliminating the linear conservation law $g_{\Psi}$, and using the result of the previous point, we end up with the formula (62).

The thermodynamic projector allows us to use almost arbitrary manifold as a quasiequilibrium closure assumption. If the projection of FPE (60) is built with the thermodynamic projector, then $\mathrm{d} S / \mathrm{d} t$ for initial system and for projected system coincide (not only the sign, but also the value). The only restriction is that the manifold must not be tangent to the level surfaces of $S$ (and must contain the equilibrium point).

Let us write down explicit formulas for the closure assumption of the form

$f(q)=\Psi^{*}(q)+\sum_{\alpha} f_{\alpha}(q) \mu_{\alpha}$.

Due to probability conservation for all $\alpha$ we have $\int f_{\alpha}(q) \mathrm{d} q=0$. Tangent spaces to the manifold (63) at all points coincide and have the form $T=\left\{\sum_{\alpha} \mu_{\alpha} f_{\alpha}(q)\right\}$. The natural coordinates in $T$ are $\mu_{\alpha}$. For every $f(q)$ of the form (63) there is the entropic scalar product, defined in $T$ :

$\langle\varphi \mid \Psi\rangle_{f}=-\left\langle\varphi \mid\left(\left.D^{2} S\right|_{f}\right) \Psi\right\rangle=\int \frac{\varphi(q) \Psi(q)}{f(q)} \mathrm{d} q$.

In the coordinates $\mu_{\alpha}$ this scalar product has the form $\left\langle\sum_{\alpha} f_{\alpha}(q) \mu_{\alpha} \mid \sum_{\beta} f_{\beta}(q) \mu^{\prime}\right\rangle_{f}=\sum_{\alpha, \beta} g_{\alpha, \beta} \mu_{\alpha} \mu_{\beta}^{\prime}$,

where

$g_{\alpha, \beta}=\int \frac{f_{\alpha}(q) f_{\beta}(q)}{f(q)} \mathrm{d} q$.

We will need the orthonormalized basis of the subspace $T \bigcap \operatorname{ker}\left(\left.D S\right|_{f}\right)$. This subspace is defined by the equation

$\int \sum_{\alpha} f_{\alpha}(q) \mu_{\alpha} \ln \frac{f(q)}{\Psi^{*}(q)} \mathrm{d} q=0$.

Let be $\int f_{1}(q) \ln \frac{f(q)}{\Psi^{*}(q)} \mathrm{d} q \neq 0$ for the definiteness. Suppose for $\alpha>1$,

$$
\begin{aligned}
q_{\alpha} & =f_{\alpha}-v_{\alpha} f_{1}, \text { where } v_{\alpha} \\
& =\frac{\int f_{\alpha}(q) \ln \left(\left(f(q) / \Psi^{*}(q)\right)\right) \mathrm{d} q}{\int f_{1}(q) \ln \left((f(q)) /\left(\Psi^{*}(q)\right)\right) \mathrm{d} q}
\end{aligned}
$$

Let us orthogonalize the family of vectors $q_{\alpha}(\alpha>1)$ with respect to the scalar product $\langle\cdot \mid \cdot\rangle_{f}$. We will get the orthogonal basis in $T \bigcap \operatorname{ker}\left(\left.D S\right|_{f}\right):\left\{e_{\alpha}\right\}(\alpha>1)$.

Let $e_{1} \in T$ be the vector, orthogonal to all $e_{\alpha}$ (for example, $\left.e_{1}=a\left(f_{1}-\sum_{\alpha>1} e_{\alpha}\left\langle f_{1} \mid e_{\alpha}\right\rangle_{f}\right)\right)$ and let $e_{1}$ be normalized in the following way: $\int e_{1}(q) \ln \left(f_{1}(q)\right) /\left(\Psi^{*}(q)\right) \mathrm{d} q=1$. The projection of the vector $J$ on $T$ is defined as:

$$
P_{f}^{\mathrm{th}} J=e_{1} \int J(q) \ln \frac{f_{1}(q)}{\Psi^{*}(q)} \mathrm{d} q+\sum_{\alpha>1} e_{\alpha} \int \frac{J(q) e_{\alpha}(q)}{f(q)} \mathrm{d} q .
$$

Projector (65) allows us to consider every manifold of the form (63) that is not tangent to the level surface of the entropy $S$, as the quasiequilibrium manifold. If the vector field is projected with the operator (65), then the dissipation is conserved.

As we can see, there is a "difficulty conservation": the solution to quasiequilibrium problem with the moment parameterization of the manifold is not explicit, and it can be difficult to calculate it. The thermodynamic projector completely eliminates this difficulty. From the other side, on the quasiequilibrium manifold with the moment parameterization (if it is found) it is easy to find the dynamics: simply write $\dot{M}_{\alpha}=\int \mu_{\alpha} J \mathrm{~d} q$. The building of the thermodynamic projector may require some efforts.

Finally, for each of the distributions $\Psi$ it is easy to find its projection on the classical quasiequilibrium manifold $\Psi \rightarrow$ $\Psi_{M(\Psi)}^{\mathrm{qe}}:$ it requires just calculation of the moments $M(\Psi)$. The analogue projection for the general thermodynamic projector is rather difficult: $\Psi \rightarrow f$ with the condition $P_{f}^{\text {th }}(\Psi-f)=$ 0 . This equation defines the projection of some neighborhood of the manifold $\Omega$ on $\Omega$, but the solution of this equation is rather difficult. Fortunately, we need to build such operators only to analyze the fast processes of the initial relaxation layer, and it is not necessary to investigate the slow dynamics. 


\section{A few words about the specifics of the computational difficulties}

From the computational point of view, the main difficulties in realization of the described methods are in the calculation of integrals of the form

$\int_{\Omega} \sum a_{i} f_{i}(q) F\left(\sum b_{j} f_{j}(q)\right) \mathrm{d} q$,

where $f_{i}$ are given functions of the vector $q, a_{i}, b_{i}$ are numbers, $F$ is a function of one variable. The usual $F$ are $F(z)=\mathrm{e}^{z} ; F(z)=1 / z$. The usual dimension of $\Omega$ in polymer physics is a few hundreds; the number of different $f_{i}$ is a few dozens. In any case, the transition from the integration of the whole FPE to solution of the moment equations gives a considerable decrease of the computation time.

In the methods of Legendre integrators and the thermodynamic projector the computational problems of linear algebra are present: the solution of the system of linear equations $C \dot{\mu}=\dot{M}$ (31), the problem of the orthogonalisation of vectors in $T_{f}$ (64) and so on. All these problems have the data that depend smoothly on the current state of $\Psi$, and, consequently, on the time $t$. So, it is possible to solve these problems with the help of perturbation theory and the methods of parametric continuation. These methods of computational linear algebra are widely used and their details are well-known. Therefore we are not discussing it here $([22,23])$.

\section{Accuracy estimation and post-processing}

Suppose that for the dynamical system (1) the approximate invariant manifold has been constructed and the slow motion equations have been derived:

$\frac{\mathrm{d} x_{s l}}{\mathrm{~d} t}=P_{x_{s l}}\left(J\left(x_{s l}\right)\right), x_{s l} \in M$,

where $P_{x_{s l}}$ is the corresponding projector onto the tangent space $T_{x_{s l}}$ of $M$. Suppose that we have solved the system (66) and have obtained $x_{s l}(t)$. Let us consider the following two questions:

- How well does this solution approximate the real solution $x(t)$ given the same initial conditions?

- How is it possible to use the solution $x_{s l}(t)$ for its refinement without solving the system (3) again?

These two questions are interconnected. The first question states the problem of the accuracy estimation. The second one states the problem of post-processing.

The simplest ("naive") estimation is given by the "invariance defect":

$\Delta_{x_{s l}}=\left(1-P_{x_{s l}}\right) J\left(x_{s l}\right)$, compared with $J\left(x_{s l}\right)$. For example, this estimation is given by $\epsilon=\left\|\Delta_{x_{s l}}\right\| /\left\|J\left(x_{s l}\right)\right\|$ using some appropriate norm.

Probably, the most comprehensive answer to this question can be given by solving the following equation:

$\frac{\mathrm{d}(\delta x)}{\mathrm{d} t}=\Delta_{x_{s l}(t)}+\left.D_{x} J(x)\right|_{s l(t)} \delta x$.

This linear equation describes the dynamics of the deviation $\delta x(t)=x(t)-x_{s l}(t)$ using the linear approximation. The solution with zero initial conditions $\delta x(0)=0$ allows estimating the robustness of $x_{s l}$ as well as the error value. Substituting $x_{s l}(t)$ for $x_{s l}(t)+\delta x(t)$ gives the required solution refinement. This dynamical post-processing [24] allows one to refine the solution substantially and to estimate its accuracy and robustness. However, the price for this is solving Eq. (68) with variable coefficients. Thus, this dynamical post-processing can be followed by a whole hierarchy of simplifications, both dynamical and static. Let us mention some of them, starting from the dynamical ones.

(1) Freezing the coefficients: In Eq. (68) the linear operator $\left.D_{x} J(x)\right|_{x_{s l}(t)}$ is replaced by its value at some distinguished point $x^{*}$ (for example, at equilibrium) or it is frozen somehow else. As a result, one gets the equation with constant coefficients and the explicit integration formula:

$\delta x(t)=\int_{0}^{t} \exp \left(D^{*}(t-\tau)\right) \Delta_{x_{s l}(\tau)} \mathrm{d} \tau$,

where $D^{*}$ is the "frozen" operator and $\delta x(0)=0$.

Another important way of freezing is substituting (68) for some model equation, i.e. substituting $D_{x} J(x)$ for $-1 / \tau^{*}$, where $\tau^{*}$ is the relaxation time. In this case the formula for $\delta x(t)$ has a very simple form:

$\delta x(t)=\int_{0}^{t} \mathrm{e}^{\tau-t \tau^{*}} \Delta_{x_{s l}(\tau)} \mathrm{d} \tau$.

(2) One-dimensional Galerkin-type approximation: Another "scalar" approximation is given by projecting (68) on $\Delta(t)=\Delta_{x_{s l}(t)}$ :

$\delta x(t)=\delta(t) \cdot \Delta(t)$,

$\frac{\mathrm{d} \delta(t)}{\mathrm{d} t}=1+\delta \frac{\langle\Delta \mid D \Delta\rangle-\langle\Delta \mid \dot{\Delta}\rangle}{\langle\Delta \mid \Delta\rangle}$,

where $\langle\mid\rangle$ is an appropriate scalar product, which can depend on the point $x_{s l}$ (for example, the entropic scalar product), $D=\left.D_{x} J(x)\right|_{s l}(t)$ or the self-adjoint linearization [17] of this operator, or some approximation of it, $\dot{\Delta}=\mathrm{d} \Delta(t) / \mathrm{d} t$.

The "hybrid" between Eqs. (71) and (68) has the simplest form (but is more difficult for computation than Eq. (71)):

$\frac{\mathrm{d}(\delta x)}{\mathrm{d} t}=\Delta(t)+\frac{\langle\Delta \mid D \Delta\rangle}{\langle\Delta \mid \Delta\rangle} \delta x$.

Here one uses the normalized matrix element $\langle\Delta \mid D \Delta\rangle /$ $\langle\Delta \mid \Delta\rangle$ instead of the linear operator $D=\left.D_{x} J(x)\right|_{x_{s l}(t)}$. 
Both Eqs. (71) and (72) can be solved explicitly:

$$
\begin{aligned}
& \delta(t)=\int_{0}^{t} \mathrm{~d} \tau \exp \left(\int_{\tau}^{t} k(\theta) \mathrm{d} \theta\right), \\
& \delta x(t)=\int_{0}^{t} \Delta(\tau) \mathrm{d} \tau \exp \left(\int_{\tau}^{t} k_{1}(\theta) \mathrm{d} \theta\right),
\end{aligned}
$$

where $k(t)=\frac{\langle\Delta \mid D \Delta\rangle-\langle\Delta \mid \dot{\Delta}\rangle}{\langle\Delta \mid \Delta\rangle}, k_{1}(t)=\frac{\langle\Delta \mid D \Delta\rangle}{\langle\Delta \mid \Delta\rangle}$.

The projection of $\Delta_{x_{s l}}(t)$ on the slow motion is zero, hence, for post-processing analysis of the slow motion, the one-dimensional model (71) should be supplemented by one more iteration:

$$
\begin{aligned}
& \frac{\mathrm{d}\left(\delta x_{s l}(t)\right)}{\mathrm{d} t}=\delta(t) P_{x_{s l}(t)}\left(D_{x} J\left(x_{s l}(t)\right)\right)(\Delta(t)), \\
& \delta x_{s l}(t)=\int_{0}^{t} \delta(\tau) P_{x_{s l}(\tau)}\left(D_{x} J\left(x_{s l}(\tau)\right)\right)(\Delta(\tau)) \mathrm{d} \tau,
\end{aligned}
$$

where $\delta(t)$ is the solution of (71).

(3) For a static post-processing one uses stationary points of dynamical Eq. (68)or their simplified versions (69), (71). Instead of Eq. (68) one gets

$$
\left.D_{x} J(x)\right|_{x_{s l}(t)} \delta x=-\Delta_{x_{s l}(t)}
$$

with one additional condition, $P_{x_{s l}} \delta x=0$. This is exactly the iteration equation of Newton's method in solving the invariance equation.

The corresponding stationary problems for the model equations and for the projections of (68) on $\Delta$ are evident. We only mention that in the projection on $\Delta$ one gets a step of the relaxation method for the invariant manifold construction. For the static post-processing with frozen parameters the "naive" estimation given by the invariance defect (67) makes sense [14].

Serious problems for reduced description can arise if the approximate invariant manifold is unstable in the sense that after small perturbations the perturbed motion can go far away. But what do these "small" and "far away" mean? There are no elaborated notions of stability for approximate invariant manifolds. Nevertheless, there exist several examples of unstable quasiequilibrium exactly invariant manifolds: explosion of the Gaussian manifold for mean-field dumbbell models in polymer dynamics [28] and simple model examples of invariant Legendre manifolds [15].

Proposed post-processing procedures can give a valid improvement of reduced model on the approximate invariant manifold if the stable invariant manifold in not far from the initial anzatz manifold. (This situation can be interpreted as a soft instability.) On the other hand, a norm of a post-processing correction can serve as a rough estimation of a distance between the initial anzatz manifold and the stable invariant manifold.

\section{Example: dumbbell model, explosion of the Gaussian anzatz and polymer stretching in flow}

Here is an example of an application of the thermodynamic projector method. In this example we consider the following simplest one-dimensional kinetic equation for the configuration distribution function $\Psi(q, t)$, where $q$ is the reduced vector connecting the beads of the dumbbell. This equation is slightly different from the FPE considered above. It is nonlinear, because of the dependence of $U$ on the moment $M_{2}[\Psi]=\int q^{2} \Psi(q) \mathrm{d} q$. This dependence allows us to get the exact quasiequilibrium equations on $M_{2}$, but these equations are not solving the problem: this quasiequilibrium manifold may become unstable when flow is present [28]. The model is:

$\partial_{t} \Psi=-\partial_{q}\{\alpha(t) q \Psi\}+\frac{1}{2} \partial_{q}^{2} \Psi$.

Here

$\alpha(t)=\kappa(t)-\frac{1}{2} f\left(M_{2}(t)\right)$,

$\kappa(t)$ is the given time-independent velocity gradient, $t$ is the reduced time, and the function $-f q$ is the reduced spring force. The function $f$ may depend on the second moment of the distribution function $M_{2}=\int q^{2} \Psi(q, t) \mathrm{d} q$. In particular, the case $f \equiv 1$ corresponds to the linear Hookean spring, while $f=\left[1-M_{2}(t) / b\right]^{-1}$ corresponds to the self-consistent finite-extension nonlinear elastic spring (the FENE-P model, first introduced in [29]). The second moment $M_{2}$ occurs in the FENE-P force $f$ as the result of the pre-averaging approximation of the original FENE model (with nonlinear spring force $f=\left[1-q^{2} / b\right]^{-1}$ ). Leading to closed constitutive equations, the FENE-P model is frequently used in simulations of complex rheological flows as the reference for more sophisticated closures to the FENE model [31-33]. The parameter $b$ changes the characteristics of the force law from Hookean at small extensions to a confining force for $q^{2} \rightarrow b$. The parameter $b$ is roughly equal to the number of monomer units represented by the dumbbell and should therefore be a large number. In the limit $b \rightarrow \infty$, the Hookean spring is recovered. Recently, it has been demonstrated that the FENE-P model appears as a first approximation within a systematic self-consistent expansion of nonlinear forces [30,14].

Eq. (77) describes an ensemble of non-interacting dumbbells subject to a pseudo-elongational flow with fixed kinematics. As is well known, the Gaussian distribution function,

$\Psi^{G}\left(M_{2}\right)=\frac{1}{\sqrt{2 \pi M_{2}}} \exp \left[-\frac{q^{2}}{2 M_{2}}\right]$

solves Eq. (77) provided the second moment $M_{2}$ satisfies

$\frac{\mathrm{d} M_{2}}{\mathrm{~d} t}=1+2 \alpha(t) M_{2}$.

Solution (79) and (80) is the valid macroscopic description if all other solutions of Eq. (77) are rapidly attracted to the family of the Gaussian distributions (79). In other words 
[34], the special solution (79) and (80) is the macroscopic description if Eq. (79) is the stable invariant manifold of the kinetic Eq. (77). If not, then the Gaussian solution is just a member of the family of solutions, and Eq. (80)has no meaning of the macroscopic equation. Thus, the complete answer to the question of validity of Eq. (80) as the macroscopic equation requires a study of dynamics in the neighborhood of the manifold Eq. (79). Because of the simplicity of the model (77), this is possible to a satisfactory level even for $M_{2}$-dependent spring forces.

In the paper [28] it was shown that there is a possibility of "explosion" of the Gaussian manifold. The qualitative description of the results of [28] is as follows: the distribution function $\Psi$ is stretched fast, but loses the Gaussian form, and after that the Gaussian form recovers slowly with the new value of $M_{2}$. For investigation of this effect the anzatz for $\Psi$ can be represented in the form:

$\Psi^{A n}(\{\sigma, \varsigma\}, q)=\frac{1}{2 \sigma \sqrt{2 \pi}}\left(\mathrm{e}^{-(q+\varsigma)^{2} / 2 \sigma^{2}}+\mathrm{e}^{-(q-\varsigma)^{2} / 2 \sigma^{2}}\right)$.

Natural inner coordinates on this manifold are $\sigma$ and $\varsigma$. Note, that now $\sigma^{2} \neq M_{2}$. The value $\sigma^{2}$ is a dispersion of one of the Gaussian summands in (81),

$M_{2}\left(\Psi^{A n}(\{\sigma, \varsigma\}, q)\right)=\sigma^{2}+\varsigma^{2}$.

To build the thermodynamic projector on the manifold (81), the thermodynamic Lyapunov function is necessary. It is necessary to emphasize that Eq. (77) is nonlinear. For such equations, the arbitrariness in the choice of the thermodynamic Lyapunov function is much smaller. Nevertheless, such a function exists. It is the free energy

$F=U\left(M_{2}[\Psi]\right)-T S[\Psi]$,

where

$S[\Psi]=-\int \Psi(\ln \Psi-1) \mathrm{d} q$,

$U\left(M_{2}[\Psi]\right)$ is the potential energy in the mean field approximation, and $T$ is the temperature (further we assume that $T=1$ ). The thermodynamic properties of the mean-field models in polymer physics are studied in the recent paper [41]

Note, that Kullback-form entropy $S_{k}=-\int \Psi \ln \left(\Psi / \Psi^{*}\right)$ also has the form $S_{k}=-F / T$ :

$\Psi^{*}=\exp (-U), \quad S_{k}[\Psi]=-\langle U\rangle-\int \Psi \ln \Psi \mathrm{d} q$.

If $U\left(M_{2}[\Psi]\right)$ in the mean-field approximation is a convex function of $M_{2}$, then the free energy (82) is a convex functional too. For the FENE-P model $U=-\ln \left[1-M_{2} / b\right]$.

In accordance with the thermodynamics the vector of flow of $\Psi$ must be proportional to the gradient of the corresponding chemical potential $\mu$ :

$J=-B(\Psi) \nabla_{q} \mu$, where $\mu=\delta F / \delta \Psi, B \geq 0$. From Eq. (82) it follows that

$$
\begin{aligned}
& \mu=\frac{\mathrm{d} U\left(M_{2}\right)}{\mathrm{d} M_{2}} q^{2}+\ln \Psi, \\
& J=-B(\Psi)\left[2 \frac{\mathrm{d} U}{\mathrm{~d} M_{2}} q+\Psi^{-1} \nabla_{q} \Psi\right] .
\end{aligned}
$$

If we suppose that $B=(D / 2) \Psi$, then we get

$$
\begin{aligned}
& J=-D\left[\frac{\mathrm{d} U}{\mathrm{~d} M_{2}} q \Psi+\frac{1}{2} \nabla_{q} \Psi\right] \\
& \frac{\partial \Psi}{\partial t}=\operatorname{div}_{q} J=D \frac{\mathrm{d} U\left(M_{2}\right)}{\mathrm{d} M_{2}} \partial_{q}(q \Psi)+\frac{D}{2} \partial^{2} q \Psi
\end{aligned}
$$

When $D=1$ these equations coincide with Eq. (77) in the absence of flow (due to Eq. (85) $\mathrm{d} F / \mathrm{d} t \leq 0$ ).

Let us construct the thermodynamic projector with the help of the thermodynamic Lyapunov function $F(82)$. The corresponding entropic scalar product at the point $\Psi$ has the form

$$
\begin{aligned}
\langle f \mid g\rangle= & \left.\frac{\mathrm{d}^{2} U}{\mathrm{~d} M_{2}^{2}}\right|_{M_{2}=M_{2}[\Psi]} \cdot \int q^{2} f(q) \mathrm{d} q \cdot \int q^{2} g(q) \mathrm{d} q \\
& +\int \frac{f(q) g(q)}{\Psi(q)} \mathrm{d} q .
\end{aligned}
$$

During the investigation of the anzatz (81) the scalar product (86), constructed for the corresponding point of the Gaussian manifold with $M_{2}=\sigma^{2}$, will be used. It will let us investigate the neighborhood of the Gaussian manifold (and to get all the results in the analytical form):

$$
\begin{aligned}
\langle f \mid g\rangle_{\sigma^{2}}= & \left.\frac{\mathrm{d}^{2} U}{\mathrm{~d} M_{2}^{2}}\right|_{M_{2}=\sigma^{2}} \cdot \int q^{2} f(q) \mathrm{d} q \cdot \int q^{2} g(q) \mathrm{d} q \\
& +\sigma \sqrt{2 \pi} \int \mathrm{e}^{q^{2} 2 \sigma^{2}} f(q) g(q) \mathrm{d} q .
\end{aligned}
$$

Also we will need to know the functional $D F$ at the point of the Gaussian manifold:

$D F_{\sigma^{2}}(f)=\left(\left.\frac{\mathrm{d} U\left(M_{2}\right)}{\mathrm{d} M_{2}}\right|_{M_{2}=\sigma^{2}}-\frac{1}{2 \sigma^{2}}\right) \int q^{2} f(q) \mathrm{d} q$,

(with the condition $\int f(q) \mathrm{d} q=0$ ). The point

$\left.\frac{\mathrm{d} U\left(M_{2}\right)}{\mathrm{d} M_{2}}\right|_{M_{2}=\sigma^{2}}=\frac{1}{2 \sigma^{2}}$,

corresponds to the equilibrium.

The tangent space to the manifold (81) is spanned by the vectors 


$$
\begin{aligned}
& f_{\sigma}=\frac{\partial \Psi^{A n}}{\partial\left(\sigma^{2}\right)}, \quad f_{\varsigma}=\frac{\partial \Psi^{A n}}{\partial\left(\varsigma^{2}\right)}, \\
& f_{\sigma}=\frac{1}{4 \sigma^{3} \sqrt{2 \pi}}\left[\mathrm{e}^{-(q+\varsigma)^{2} 2 \sigma^{2}} \frac{(q+\varsigma)^{2}-\sigma^{2}}{\sigma^{2}}\right. \\
&\left.+\mathrm{e}^{-(q-\varsigma)^{2} 2 \sigma^{2}} \frac{(q-\varsigma)^{2}-\sigma^{2}}{\sigma^{2}}\right], \\
& f_{\varsigma}=\frac{1}{4 \sigma^{2} \varsigma \sqrt{2 \pi}}\left[-\mathrm{e}^{-(q+\varsigma)^{2} 2 \sigma^{2}} \frac{q+\varsigma}{\sigma}+\mathrm{e}^{-(q-\varsigma)^{2} 2 \sigma^{2}} \frac{(q-\varsigma)}{\sigma}\right] .
\end{aligned}
$$

The Gaussian entropy (free energy) production in the directions $f_{\sigma}$ and $f_{\varsigma}(88)$ has a very simple form:

$D F_{\sigma^{2}}\left(f_{\varsigma}\right)=D F_{\sigma^{2}}\left(f_{\sigma}\right)=\left.\frac{\mathrm{d} U\left(M_{2}\right)}{\mathrm{d} M_{2}}\right|_{M_{2}=\sigma^{2}}-\frac{1}{2 \sigma^{2}}$.

The linear subspace $\operatorname{ker} D F_{\sigma^{2}}$ in $\operatorname{lin}\left\{f_{\sigma}, f_{\varsigma}\right\}$ is spanned by the vector $f_{\varsigma}-f_{\sigma}$.

Let us have the given vector field $\mathrm{d} \Psi / \mathrm{d} t=\Phi(\Psi)$ at the point $\Psi(\{\sigma, \varsigma\})$. We need to build the projection of $\Phi$ onto the tangent space $T_{\sigma, \varsigma}$ at the point $\Psi(\{\sigma, \varsigma\})$ :

$P_{\sigma, \zeta}^{\text {th }}(\Phi)=\varphi_{\sigma} f_{\sigma}+\varphi_{\varsigma} f_{\varsigma}$.

This equation implies that the equations for $\sigma^{2}$ and $\varsigma^{2}$ will have the forms

$\frac{\mathrm{d} \sigma^{2}}{\mathrm{~d} t}=\varphi_{\sigma}, \frac{\mathrm{d} \varsigma^{2}}{\mathrm{~d} t}=\varphi_{\varsigma}$.

Projection $\left(\varphi_{\sigma}, \varphi_{\varsigma}\right)$ can be found from the following two equations:

$\varphi_{\sigma}+\varphi_{\varsigma}=\int q^{2} \Phi(\Psi)(q) \mathrm{d} q$

$\left\langle\varphi_{\sigma} f_{\sigma}+\varphi_{\varsigma} f_{\zeta} \mid f_{\sigma}-f_{\zeta}\right\rangle_{\sigma^{2}}=\left\langle\Phi(\Psi) \mid f_{\sigma}-f_{\zeta}\right\rangle_{\sigma^{2}}$,

where $\langle f \mid g\rangle_{\sigma^{2}}=\left\langle\Phi(\Psi) \mid f_{\sigma}-f_{\varsigma}\right\rangle_{\sigma^{2}}$, (86). The first equation in (93) implies that the time derivative $\mathrm{d} M_{2} / \mathrm{d} t$ is the same for the initial and the reduced equations. Due to the formula for the dissipation of the free energy (88), this equality is equivalent to the persistence of the dissipation in the neighborhood of the Gaussian manifold. The second equation in (93) means that $\Phi$ is projected orthogonally on ker $D S \bigcap T_{\sigma, \varsigma}$. Let us use the orthogonality with respect to the entropic scalar product (87). The solution of Eq. (93) has the form where $\Phi=\Phi(\Psi), M_{2}(\Phi)=\int q^{2} \Phi(\Psi) \mathrm{d} q$.

It is easy to check that the formulas (94) are indeed defining the projector: if $f_{\sigma}$ (or $f_{\varsigma}$ ) is substituted there instead of the function $\Phi$, then we will get $\varphi_{\sigma}=1, \varphi_{\varsigma}=0$ (or $\varphi_{\sigma}=0, \varphi_{\varsigma}=1$, respectively). Let us substitute the right part of the initial kinetic Eq. (77), calculated at the point $\Psi(q)=\Psi(\{\sigma, \varsigma\}, q)$ (see Eq. (81)) in Eq. (94) instead of $\Phi$. We will get the closed system of equations on $\sigma^{2}, \varsigma^{2}$ in the neighborhood of the Gaussian manifold.

This system describes the dynamics of the distribution function $\Psi$. The distribution function is represented as the half-sum of two Gaussian distributions with the averages of distribution $\pm \varsigma$ and mean-square deviations $\sigma$. All integrals in the right-hand-part of Eq. (94) are possible to calculate analytically.

Basis $\left(f_{\sigma}, f_{\varsigma}\right)$ is convenient to use everywhere, except the points in the Gaussian manifold, $\varsigma=0$, because if $\varsigma \rightarrow 0$, then

$f_{\sigma}-f_{\varsigma}=O\left(\frac{\varsigma^{2}}{\sigma^{2}}\right) \rightarrow 0$.

To analyze the relaxation in the small neighborhood of the Gaussian manifold it is more convenient to use another basis:

$F^{+}=f_{\sigma}+f_{\zeta} ; F^{+}=\frac{\sigma^{2}}{\varsigma^{2}}\left(f_{\sigma}-f_{\zeta}\right)$.

This corresponds to a reparametrization of the initial manifold (81):

$$
\begin{aligned}
\Psi(\{\xi, \varsigma\}, q)= & \frac{1}{2 \sqrt{2 \pi} \sqrt{\xi^{2}-\varsigma^{2}}} \\
& \times\left(\mathrm{e}^{-(q+\varsigma)^{2} / 2\left(\xi^{2}-\varsigma^{2}\right)}+\mathrm{e}^{-(q-\varsigma)^{2} / 2\left(\xi^{2}-\varsigma^{2}\right)}\right) .
\end{aligned}
$$

Let us analyze the stability of the Gaussian manifold to the "dissociation" of the Gaussian peak in two peaks (81). To do this, it is necessary to find the first non-zero term in the Taylor expansion in $\varsigma^{2}$ of the right-hand-side of the second equation in the system (94). The denominator has the order of $\varsigma^{4}$, the numerator has, as it is easy to see, the order not less than $\varsigma^{6}$ (because the Gaussian manifold is invariant with respect to the initial system).

Let us denote $G_{\sigma}=\frac{1}{\sqrt{2 \pi}} \mathrm{e}^{-q^{2} / \sigma^{2}}$. Then we get

$$
\begin{aligned}
& \Psi(\{\sigma, \varsigma\}, q)=G_{\sigma}(q)\left[1+\frac{1}{2} \frac{\varsigma^{2}}{\sigma^{2}}\left(\frac{q^{2}}{\sigma^{2}}-1\right)+\frac{1}{4} \frac{\varsigma^{4}}{\sigma^{4}}\left(\frac{1}{2}-\frac{q^{2}}{\sigma^{2}}+\frac{1}{6} \frac{q^{4}}{\sigma^{4}}\right)\right]+o\left(\frac{\varsigma^{4}}{\sigma^{4}}\right) \\
& f_{\sigma}=\frac{G_{\sigma}(q)}{2 \sigma^{2}}\left[\frac{q^{2}}{\sigma^{2}}-1+\frac{\varsigma^{2}}{\sigma^{2}}\left(\frac{1}{2} \frac{q^{4}}{\sigma^{4}}-3 \frac{q^{2}}{\sigma^{2}}+\frac{3}{2}\right)+\frac{\varsigma^{4}}{\sigma^{4}}\left(\frac{1}{24} \frac{q^{6}}{\sigma^{6}}-\frac{15}{24} \frac{q^{4}}{\sigma^{4}}+\frac{15}{8} \frac{q^{2}}{\sigma^{2}}-\frac{5}{8}\right)\right]+o\left(\frac{\varsigma^{4}}{\sigma^{4}}\right)
\end{aligned}
$$

$$
\frac{\mathrm{d} \sigma^{2}}{\mathrm{~d} t}=\varphi_{\sigma}=\frac{\left\langle\Phi \mid f_{\sigma}-f_{\varsigma}\right\rangle_{\sigma^{2}}+M_{2}(\Phi)\left(\left\langle f_{\varsigma} \mid f_{\zeta}\right\rangle_{\sigma^{2}}-\left\langle f_{\sigma} \mid f_{\varsigma}\right\rangle_{\sigma^{2}}\right)}{\left\langle f_{\sigma}-f_{\varsigma} \mid f_{\sigma}-f_{\varsigma}\right\rangle_{\sigma^{2}}}, \quad \frac{\mathrm{d} \varsigma^{2}}{\mathrm{~d} t}=\varphi_{\varsigma}=\frac{-\left\langle\Phi \mid f_{\sigma}-f_{\varsigma}\right\rangle_{\sigma^{2}}+M_{2}(\Phi)\left(\left\langle f_{\sigma} \mid f_{\sigma}\right\rangle_{\sigma^{2}}-\left\langle f_{\sigma} \mid f_{\varsigma}\right\rangle_{\sigma^{2}}\right)}{\left\langle f_{\sigma}-f_{\varsigma} \mid f_{\sigma}-f_{\varsigma}\right\rangle_{\sigma^{2}}}
$$




$$
\begin{aligned}
& f_{\varsigma}=\frac{G_{\sigma}(q)}{2 \sigma^{2}}\left[\frac{q^{2}}{\sigma^{2}}-1+\frac{\varsigma^{2}}{\sigma^{2}}\left(\frac{1}{6} \frac{q^{4}}{\sigma^{4}}-\frac{q^{2}}{\sigma^{2}}+\frac{1}{2}\right)+\frac{\varsigma^{4}}{\sigma^{4}}\left(\frac{1}{120} \frac{q^{6}}{\sigma^{6}}-\frac{1}{8} \frac{q^{4}}{\sigma^{4}}+\frac{3}{8} \frac{q^{2}}{\sigma^{2}}-\frac{1}{8}\right)\right]+o\left(\frac{\varsigma^{4}}{\sigma^{4}}\right) ; \\
& f_{\sigma}-f_{\varsigma}=\frac{\varsigma^{2}}{\sigma^{2}} \frac{1}{2 \sigma^{2}} G_{\sigma}(q)\left[\frac{1}{3} \frac{q^{4}}{\sigma^{4}}-2 \frac{q^{2}}{\sigma^{2}}+1+\frac{\varsigma^{2}}{\sigma^{2}}\left(\frac{1}{30} \frac{q^{6}}{\sigma^{6}}-\frac{1}{2} \frac{q^{4}}{\sigma^{4}}+\frac{3}{2} \frac{q^{2}}{\sigma^{2}}-\frac{1}{2}\right)\right]+o\left(\frac{\varsigma^{4}}{\sigma^{4}}\right) .
\end{aligned}
$$

Let us calculate $\partial_{t} \Psi=\Phi(\Psi(\{\sigma, \varsigma\}))$ with the accuracy up to $\varsigma^{4}$ :

$$
\begin{aligned}
& \frac{1}{2} \partial_{q}^{2} \Psi(\{\sigma, \varsigma\})=f_{\sigma} ; \quad M_{2}\left(\frac{1}{2} \partial_{q}^{2} \Psi(\{\sigma, \varsigma\})\right)=1 ; \quad M_{2}(\Psi(\{\sigma, \varsigma\}))=\sigma^{2}+\varsigma^{2} \\
& -\alpha \partial_{q}(q \Psi(\{\sigma, \varsigma\}))=\alpha G_{\sigma}(q)\left[\frac{q^{2}}{\sigma^{2}}-1+\frac{\varsigma^{2}}{\sigma^{2}}\left(\frac{1}{2} \frac{q^{4}}{\sigma^{4}}-2 \frac{q^{2}}{\sigma^{2}}+\frac{1}{2}\right)+\quad \frac{\varsigma^{4}}{\sigma^{4}}\left(\frac{1}{24} \frac{q^{6}}{\sigma^{6}}-\frac{11}{24} \frac{q^{4}}{\sigma^{4}}+\frac{7}{8} \frac{q^{2}}{\sigma^{2}}-\frac{1}{8}\right)\right]+o\left(\frac{\varsigma^{4}}{\sigma^{4}}\right) ;
\end{aligned}
$$

$M_{2}\left(-\alpha \partial_{q}(q \Psi(\{\sigma, \varsigma\}))\right)=2 \alpha\left(\sigma^{2}+\varsigma^{2}\right)+o\left(\frac{\varsigma^{4}}{\sigma^{4}}\right)$.

The diffusion part gives the zero contribution to the numerator of Eq. (94):

$-\left\langle f_{\sigma} \mid f_{\sigma}-f_{\varsigma}\right\rangle+\left\langle f_{\sigma} \mid f_{\sigma}-f_{\varsigma}\right\rangle=0$.

Therefore, to find $\mathrm{d} \zeta / \mathrm{d} t$ it is sufficient to use $\Phi_{1}=$ $-\alpha \partial_{q}(q \Psi)$, so we get

$$
\begin{aligned}
& M_{2}\left(\Phi_{1}(\Psi(\{\sigma, \varsigma\}))\right) f_{\sigma}-\Phi_{1}(\Psi(\{\sigma, \varsigma\})) \\
& \quad=\alpha G_{\sigma}(q) \frac{\varsigma^{4}}{\sigma^{4}}\left(\frac{1}{3} \frac{q^{4}}{\sigma^{4}}-2 \frac{q^{2}}{\sigma^{2}}+1\right)+o\left(\frac{\varsigma^{4}}{\sigma^{4}}\right) \\
& =2 \alpha \sigma^{2} \frac{\varsigma^{2}}{\sigma^{2}}\left(f_{\sigma}-f_{\varsigma}\right)+o\left(\frac{\varsigma^{4}}{\sigma^{4}}\right) .
\end{aligned}
$$

Thus

$$
\frac{1}{\sigma^{2}} \frac{\mathrm{d} \varsigma^{2}}{\mathrm{~d} t}=2 \alpha \frac{\varsigma^{2}}{\sigma^{2}}+o\left(\frac{\varsigma^{4}}{\sigma^{4}}\right) .
$$

So, if $\alpha>0$, then $\varsigma^{2}$ grows exponentially $\left(\varsigma \sim \mathrm{e}^{\alpha t}\right)$ and the Gaussian manifold is unstable; if $\alpha<0$, then $\varsigma^{2}$ decreases exponentially and the Gaussian manifold is stable.

The form of the phase trajectories is shown qualitative in Fig. 1

The exact analytical computation for nonlinear case (94) leads to exactly the same result without $o(\ldots): \mathrm{d} \varsigma^{2} / \mathrm{d} t=$ $2 \alpha \varsigma^{2}, \mathrm{~d} \sigma^{2} / \mathrm{d} t=1+2 \alpha \sigma^{2}$.

For the real FPE (for example, with the FENE potential) the motion in the presence of flow can be represented as

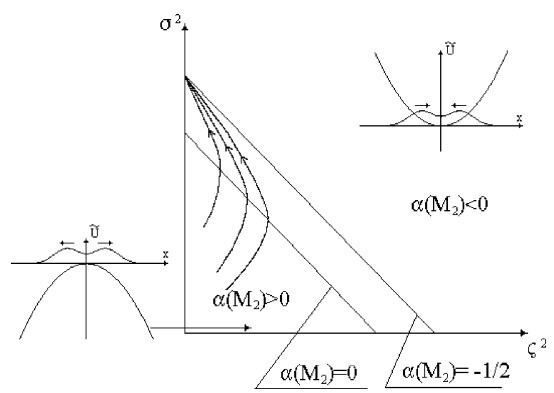

Fig. 1. Phase trajectories for two-peak approximation, FENE-P model. The vertical axis $(\varsigma=0)$ corresponds to the Gaussian manifold. The triangle with $\alpha\left(M_{2}\right)>0$ is the domain of exponential instability. the motion in the effective potential well $\tilde{U}(q)=U(q)-$ $\kappa q^{2}$. Different variants of the phase portrait for the FENE potential are presented in Fig. 2. Instability and dissociation of the unimodal distribution functions ("peaks") for the FPE is the general effect when flow is present. The instability occurs when the matrix $\partial^{2} \tilde{U} / \partial q_{i} \partial q_{j}$ starts to have negative eigenvalues $(\tilde{U}$ is the effective potential energy, $\tilde{U}(q)=$ $\left.U(q)-\sum_{i, j} \kappa_{i, j} q_{i} q_{j}\right)$.

The stationary polymodal distribution corresponds to the persistence of several local minima of the function $\tilde{U}(q)$. The multidimensional case is different from the one-dimensional case because it has a huge amount of possible configurations. All normal forms of the catastrophe of "birth of the critical point" are well investigated and known [42]. Every dissociation of the peak is connected with such a catastrophe. The number of new peaks is equal to the number of the new local minima of $U$.

It is not very difficult to perform the analysis of Eqs. (94) for every quantity of peaks and every potential. Moreover, for the polynomial potentials all the necessary integrals are possible to calculate analytically (if the coefficients of the scalar product and entropy production are taken in the Gaussian point). The same situation also applies to the general $n$-dimensional Gaussian distributions:

$G_{\xi, \Sigma}=A \exp \left(-\frac{1}{2} \sum_{i, j}\left(\Sigma^{-1}\right)_{i j}\left(q_{i}-\xi_{i}\right)\left(q_{j}-\xi_{j}\right)\right)$,

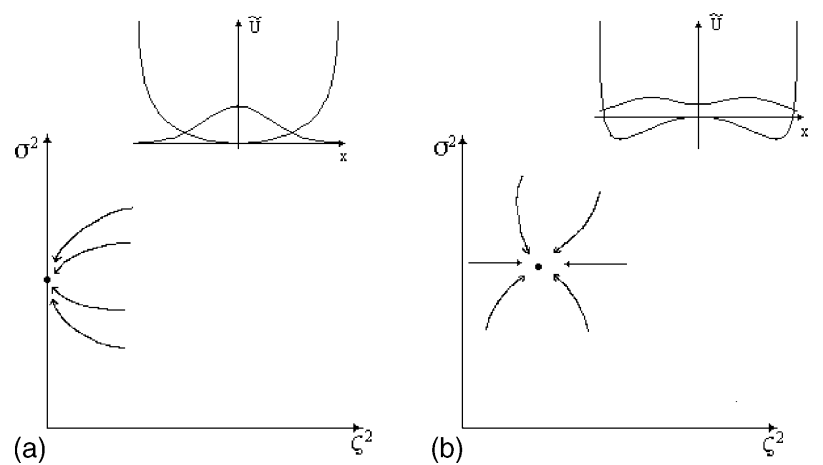

Fig. 2. Phase trajectories for two-peak approximation, FENE model: (a) a stable equilibrium on the vertical axis, one stable peak and (b) a stable equilibrium with $\varsigma>0$, stable two-peak configuration. 
where $\Sigma$ is the covariance matrix, $\xi_{i}=\left\langle q_{i}\right\rangle$ is the expectation of $q_{i}$. The normalization constant $A$ is

$A=\left((2 \pi)^{n} \operatorname{det} \Sigma\right)^{1 / 2}$.

Here in the equation for the effective energy we have the symmetric part of the tensor $\kappa_{i j}=\partial^{2} U / \partial q_{i} \partial q_{j}$. The presence of the asymmetric part may lead to the relaxation oscillations (both for the FPE and for the peak dynamics).

For the modeling of dynamics of the multimodal distributions for FPE with the presence of the flow (the flow may be nonstationary) it seems to be useful to use the physically clear modeling of the distribution function as a sum of the finite number of the Gaussian peaks. Thermodynamic projector gives us an opportunity to make this models thermodynamically consistent.

\section{The thermodynamic projector and post-processing for Gaussian manifolds}

Multidimensional Gaussian distributions (97) form a manifold of distribution functions. It is natural to use this manifold as a first (and simplest) anzatz for model reduction. More complicated approximations for distribution functions should be considered, if this Gaussian anzatz is insufficient for modeling, for example, for systems with instabilities.

For the FPE with polynomial potential $U(q)$ the thermodynamic projector and some of post-processing procedures for the Gaussian anzatz do not require numerical calculation of multidimensional integrals mentioned in Section 9. These integrals can be calculated analytically: for any dimension $n$ and any polynomial $P(q)$ the integral $\int P(q) G_{\xi, \Sigma}(q) \mathrm{d}^{n} q$ can be calculated by using the bosonic Wick formula [44,45]: let $X_{1}, \ldots, X_{m}$ be random variables and have a joint Gaussian distribution; then the mathematical expectation of the product of $X_{i}$ is

$$
\begin{aligned}
E\left(\prod_{i=1}^{m} X_{i}\right) & =0, \quad \text { if } m \text { is odd; } E\left(\prod_{i=1}^{m} X_{i}\right) \\
& =\sum_{I, J} c_{i_{1} j_{1}} c_{i_{2} j_{2}}, \ldots, c_{i_{k} j_{k}},
\end{aligned}
$$

if $m=2 k$, where the sum runs over all partition of $\{1,2, \ldots, 2 k\}=I \cup J, I=\left\{i_{r}\right\}_{1}^{k}, J=\left\{j_{r}\right\}_{1}^{k}$, such that $i_{1}<\cdots<i_{k}, j_{1}<\cdots<j_{k}$ and for each $r i_{r}<j_{r}$

Let us describe the thermodynamic projector and one-dimensional post-processing for multidimensional Gaussian anzatz. The Gaussian distribution (97) is the solution for optimization problem:

$$
S_{0}(\Psi) \rightarrow \max , \quad m_{i}(\Psi)=\xi_{i}, \quad \sigma_{i j}(\Psi)=\Sigma_{i j},
$$

where $m_{i}(\Psi)=\int q_{i} \Psi(q) \mathrm{d}^{n} q, \sigma_{i j}(\Psi)=\int\left(q_{i}-\xi_{i}\right)\left(q_{j}-\right.$ $\left.\xi_{j}\right) \Psi(q) \mathrm{d}^{n} q$. The entropy $S_{0}$ here is the classical Boltzmann entropy: $S_{0}=\int \Psi(q)(\ln \Psi(q)-1) \mathrm{d}^{n} q$. This entropy is not a Lyapunov function for the FPE. The corresponding Lyapunov function is Kullback-form entropy

$$
\begin{aligned}
S & =-\int \Psi(q)\left(\ln \left(\frac{\Psi(q)}{\Psi^{*}(q)}\right)-1\right) \mathrm{d}^{n} q \\
& =S_{0}-\int U(q) \Psi(q) \mathrm{d}^{n} q .
\end{aligned}
$$

It has the form $S=-F$, where $F$ is the free energy. (The temperature $T$ is normalized to 1.) The second differential for the classical entropy (100) and for the Kullback-form entropy (101) is the same, hence, the entropic scalar product is also the same: $\langle f(q) \mid g(q)\rangle_{\Psi}=\int f(q) g(q) / \Psi(q) \mathrm{d}^{n} q$. Therefore the orthogonal projector onto the tangent space to the Gaussian manifold can be written through moment parameterization, in the same way as for a quasiequilibrium manifold:

$$
\begin{aligned}
P^{\perp}(J(q))= & \sum_{i} \frac{\partial G_{\xi, \Sigma}}{\partial \xi_{i}} m_{i}(J(q))+\sum_{i j} \frac{\partial G_{\xi, \Sigma}}{\partial \Sigma_{i j}} m_{i j}^{2}(J(q)) \\
& -\xi_{i} m_{j}(J(q))-\xi_{j} m_{i}(J(q)),
\end{aligned}
$$

where $m_{i}(J(q))=\int q_{i} J(q) \mathrm{d}^{n} q, m_{i j}^{2}(J(q))=\int q_{i} q_{j} J(q) \mathrm{d}^{n} q$ This formula for orthogonal projection of $J$ (102) follows from usual chain rule and from moment equations: if $\dot{\Psi}=J$, then $\dot{\xi}=m(J)$ and $\dot{\Sigma}_{i j}=m_{i j}^{2}(J(q))-\xi_{i} m_{j}(J(q))-$ $\xi_{j} m_{i}(J(q))$.

The gradients of $S(\Psi)(100)$ and $S_{0}(\Psi)$ (101) with respect to the entropic scalar product in point $\Psi$ are:

$$
\begin{gathered}
\operatorname{grad} S_{0}=-\Psi(q)\left(\ln (\Psi(q))-\int \Psi\left(q^{\prime}\right) \ln \left(\Psi\left(q^{\prime}\right)\right) \mathrm{d}^{n} q^{\prime}\right), \\
\operatorname{grad} S=\operatorname{grad} S_{0}-\Psi(q)\left(U(q)-\int \Psi\left(q^{\prime}\right) U\left(q^{\prime}\right) \mathrm{d}^{n} q^{\prime}\right) .
\end{gathered}
$$

Integrals in right-hand-parts of Eqs. (103) provide conditions of zero means:

$\int \operatorname{grad} S_{0}(q) \mathrm{d}^{n} q=\int \operatorname{grad} S(q) \mathrm{d}^{n} q=0$.

These conditions are necessary, because functionals $D S_{0}, D S$ act on differences of distribution functions that have zero mean values. The gradient grad $S_{0}$ is tangent to the Gaussian manifold at the point $\Psi$, because this manifold is quasiequilibrium with respect to the entropy $S_{0}$. Let us use the orthogonal projector (102) to split grad $S$ (103) onto tangent and orthogonal components:

$\operatorname{grad} S=\operatorname{grad} S^{\|}+\operatorname{grad} S^{\perp}=\left(1-P^{\perp}\right) \operatorname{grad} S+P^{\perp} \operatorname{grad} S$.

The thermodynamic projector $P^{\text {th }}$ has the form

$P^{\text {th }} J=P^{\perp} J+\frac{\operatorname{grad} S^{\|}}{\left\langle\operatorname{grad} S^{\|} \mid \operatorname{grad} S^{\|}\right\rangle}\left\langle\operatorname{grad} S^{\perp} \mid J\right\rangle$.

It is important to mention that the second term of the thermodynamic projector (104) becomes singular if $\operatorname{grad} S^{\|}=$ 0 . These points are the stationary points of the entropy $S$ 
on the Gaussian manifold. These singularities provide the preservation of entropy production near these points. The singularity of projection has a transparent physical sense. The relaxation along the Gaussian manifold to the point $\Psi_{\mathrm{G}}^{*}$ of entropy maximum on this manifold is not complete, because this point is not the equilibrium. This motion should be rated as a step of relaxation, and after it was completed, the next step should start. In this sense it is obvious that the motion to the point $\Psi_{\mathrm{G}}^{*}$ along the anzatz manifold should take the finite time. The results of this step-by-step relaxation can represent the whole process (with smoothing [46], or without it [47]). The experience of such step-by-step computing of relaxation trajectories in the initial layer problem for the Boltzmann kinetics demonstrated it's efficiency [46,47].

Let us project the right-hand-part of the FPE (34) onto the tangent space to the Gaussian manifold. For polynomial potentials both the projection and the invariance defect $\Delta$ can be calculated analytically. For one-dimensional post-processing procedure (71) one needs the matrix element $\langle\Delta \mid D \Delta\rangle$ and the scalar products $\langle\Delta \mid \dot{\Delta}\rangle,\langle\Delta \mid \Delta\rangle$. Here $D$ is the linear operator of FPE. Calculation of all these scalar products does not need the numerical integration in multidimensional space. The same is truth also for a supplementary operation (75). So, the thermodynamic projection of the FPE with polynomial potential onto the Gaussian manifold and one-dimensional postprocessing of solutions for this projected equation can be performed using the bosonic Wick formula without numerical integration into the configuration space.

\section{Conclusion}

In this work we presented a toolbox for the development and reduction of dynamical models of nonequilibrium systems with the persistence of the correct dissipation. The basic notions of this toolbox are entropy, quasiequilibrium (MaxEnt) distribution, dual variables, and the thermodynamic projector. The main technical ideas are: Legendre integrators, dynamical post-processing, and the transformation of almost any arbitrary anzatz into a thermodynamically consistent model via the thermodynamic projector.

The Legendre integrators are based on a simple, but very useful idea: to write and solve dynamic equations for dual variables. This idea is efficient, because to obtain the dynamic equations for dual variables it is necessary to solve linear equations. To get the usual quasiequilibrium dynamical equations for the moments, we should solve nonlinear (transcendent) equations. Sometimes it happens that these equations can be written down in explicit form (Vlasov equation, Euler equation, 10moments Gaussian approximation in gas kinetics [6,43]), but usually these equations remain in implicit form with right-hand-sides derived by a system of transcendent equations.
The post-processing is necessary for accuracy estimation. It gives us the cheapest way to improve the solution obtained by the Legendre integrators.

The thermodynamic projector allows one to transform almost any arbitrary anzatz into a physically consistent dynamic model with persistence of dissipation. The simplest example, discussed in details, is the two peaks model for Gaussian manifold instability in polymer dynamics. This type of models opens a way to create the computational models for the "molecular individualism" [48-50].

The simplest model of molecular individualism is the "Gaussian parallelepiped". The distribution function is represented as a sum of $2^{m}$ Gaussian peaks located at the vertices of a centrally symmetrical parallelepiped:

$$
\begin{aligned}
\Psi(q)= & 1 / 2^{m}(2 \pi)^{n / 2} \sqrt{\operatorname{det} \Sigma} \sum_{\varepsilon_{i}= \pm 1,(i=1, \ldots, m)} \\
& \times \exp \left(-\frac{1}{2}\left(\Sigma^{-1}\left(q+\sum_{i=1}^{m} \varepsilon_{i} \varsigma_{i}\right), q+\sum_{i=1}^{m} \varepsilon_{i} \varsigma_{i}\right)\right),
\end{aligned}
$$

where $n$ is the dimension of configuration space, $2 \varsigma_{i}$ is the vector of the $i$ th edge of the parallelepiped, and $\Sigma$ is the one peak covariance matrix (in this model $\Sigma$ is the same for all peaks). The macroscopic variables for this model are:

(1) The covariance matrix $\Sigma$.

(2) The set of vectors $\varsigma_{i}$ (or the parallelepiped edges).

The dimension is $n(n+1) / 2+m n$.

The number $m(m \leq n)$ is the estimated number of nonstable directions of motion (dimension of instability). To include the non-Gaussian equilibrium the "Gaussian parallelepiped" should be deformed to non-Gaussian "peaks parallelepiped". Technical details will be discussed in the separate paper. The structure of "peaks parallelepiped" leads to the molecular individualism in such a way: each individual molecule belongs to a domain of a peak in configuration space. The number of these peaks grows significantly with the dimension of instability, as $2^{m}$, and even if $m=3$, then the number of peaks is 8 , and one should discover 8 distinguished sorts of molecular configurations. On the other hand, in projection on a line this amount of peaks can form a distribution without a clue about peak structure, hence, the study of properties of ensembles (viscosity, stress coefficient, etc.) can be without any hint to a cluster structure in configuration space.

\section{Acknowledgements}

The authors are grateful to Dr. P. Ilg and Professor H.C. Öttinger for fruitful collaboration and valuable discussion.

\section{References}

[1] G.W. Gibbs, Elementary Principles of Statistical Mechanics, Dover, 1960. 
[2] E.T. Jaynes, Information theory and statistical mechanics, in: Statistical Physics, Brandeis Lectures, vol. 3, 1963, pp. 160-185.

[3] D. Zubarev, V. Morozov, G. Röpke, Statistical Mechanics of Nonequilibrium Processes, vol. 1, Basic Concepts, Kinetic Theory, Akademie, Verlag, Berlin, 1996; vol. 2, Relaxation and Hydrodynamic Processes, Akademie, Verlag, Berlin, 1997.

[4] H. Grad, On the kinetic theory of rarefied gases, Comm. Pure Appl. Math. 2 (4) (1949) 331-407.

[5] A.M. Kogan, L.I. Rozonoer, On the macroscopic description of kinetic processes, Dokl. AN SSSR 158 (3) (1964) 566-569.

[6] A.M. Kogan, Derivation of Grad-type equations and study of their properties by the method of entropy maximization, Prikl. Math. Mech. 29 (1) (1965) 122-133.

[7] L.I. Rozonoer, Thermodynamics of nonequilibrium processes far from equilibrium, in: Thermodynamics and Kinetics of Biological Processes, Nauka, Moscow, 1980, pp. 169-186.

[8] A.N. Gorban, Equilibrium Encircling. Equations of Chemical Kinetics and Their Thermodynamic Analysis, Nauka, Novosibirsk, 1984.

[9] J. Karkheck, G. Stell, Maximization of entropy, kinetic equations, and irreversible thermodynamics, Phys. Rev. A 25 (6) (1984) 33023327.

[10] N.N. Bugaenko, A.N. Gorban, I.V. Karlin, Universal Expansion of the Triplet Distribution Function, Teoreticheskaya i Matematicheskaya Fisika 88 (3) (1991) 430-441 (English Translation: Theoret. Math. Phys. (1992) 977-985).

[11] R. Balian, Y. Alhassid, H. Reinhardt, Dissipation in many-body systems: a geometric approach based on information theory, Phys. Rep. 131 (1) (1986) 1-146.

[12] P. Ilg, I.V. Karlin, H.C. Öttinger, Canonical distribution functions in polymer dynamics: I. Dilute solutions of flexible polymers, Physica A 315 (2002) 367-385.

[13] P. Ilg, I.V. Karlin, M. Kröger, H.C. Öttinger, Canonical distribution functions in polymer dynamics: II. Liquid-crystalline polymers, Physica A 319 (2003) 134-150.

[14] A.N. Gorban, I.V. Karlin, P. Ilg, H.C. Öttinger, Corrections and enhancements of quasi-equilibrium states, J. Non-Newtonian Fluid Mech. 96 (2001) 203-219.

[15] M. Grmela, Reciprocity relations in thermodynamics, Physica A 309 (2002) 304-328.

[16] A.N. Gorban, I.V. Karlin, Thermodynamic parameterization, Physica A 190 (1992) 393-404.

[17] A.N. Gorban, I.V. Karlin, Method of invariant manifold for chemical kinetics, Chem. Eng. Sci. 58 (21) (2003) 4751-4768. Online: arXiv: cond-mat/0207231.

[18] A.N. Gorban, I.V. Karlin, Family of additive entropy functions out of thermodynamic limit, Phys. Rev. E 67 (2003) 016104. Online: arXiv: cond-mat/0205511.

[19] A.N. Gorban, I.V. Karlin, H.C. Öttinger, The additive generalization of the Boltzmann entropy, Phys. Rev. E 67 (2003) 067104. Online: arXiv: cond-mat/0209319.

[20] P. Gorban, Monotonically equivalent entropies and solution of additivity equation, Physica A 328 (3-4) (2003) 380-390. Online: arXiv: cond-mat/0304131.

[21] S. Abe, Y. Okamoto (Eds.), Nonextensive Statistical Mechanics and its Applications, Springer, Heidelberg, 2001.

[22] E.L. Allgower, K. Georg, Numerical Continuation Methods, Springer, Berlin, 1990

[23] J.W. Demmel, Applied Numerical Linear Algebra, SIAM, Philadelphia, 1997.

[24] L.G. Margolin, E.S. Titi, S. Wynne, The postprocessing Galerkin and nonlinear Galerkin methods-a truncation analysis point of view, SIAM J. Numer. Anal. 41 (2) (2003) 695-714.

[25] K. Gustafsson, Control theoretic techniques for stepsize selection in implicit Runge-Kutta methods, ACM Trans. Math. Software 20 (1994) 496-517.
[26] E. Hairer, S.P. Norsett, G. Wanner, Solving ordinary differential equations I nonstiff problems, Springer Series in Computational Mathematics, vol. 8, Springer-Verlag, 1987.

[27] E. Hairer, G. Wanner, Solving ordinary differential equations II stiff and differential-algebraic problems, Springer Series in Computational Mathematics, vol. 14, Springer-Verlag, 1991.

[28] P. Ilg, I.V. Karlin, Validity of macroscopic description in dilute polymeric solutions, Phys. Rev. E 62 (2000) 1441-1443

[29] R.B. Bird, R.B. Dotson, N.J. Jonson, Polymer solution rheology based on a finitely extensible bead-spring chain model, J. Non-Newtonian Fluid Mech. 7 (1980) 213-235; Corrigendum 8 (1981) 193.

[30] P. Ilg, I.V. Karlin, H.C. Öttinger, Generating moment equations in the Doi model of liquid-crystalline polymers, Phys. Rev. E 60 (1999) 5783-5787.

[31] H.C. Öttinger, Stochastic Processes in Polymeric Fluids, Springer, Berlin, 1996.

[32] G. Lielens, P. Halin, I. Jaumin, R. Keunings, V. Legat, New closure approximations for the kinetic theory of finitely extensible dumbbells, J. Non-Newtonian Fluid Mech. 76 (1998) 249-279.

[33] M. Kröger, Simple models for complex nonequilibrium fluids, Phys. Rep. 390 (6) (2004) 453-551.

[34] A.N. Gorban, I.V. Karlin, Method of invariant manifolds and regularization of acoustic spectra, Transport Theory Stat. Phys. 23 (1994) 559-632.

[35] V.B. Zmievskii, I.V. Karlin, M. Deville, The universal limit in dynamics of dilute polymeric solutions, Physica A 275 (1-2) (2000) 152-177.

[36] D. Jou, J. Casas-Vázquez, G. Lebon, Extended Irreversible Thermodynamics, Springer, Berlin, 1993.

[37] A.N. Gorban, I.V. Karlin, Scattering rates versus moments: alternative Grad equations, Phys. Rev. E 54 (1996) R3109.

[38] A.N. Gorban, I.V. Karlin, Quasi-equilibrium approximation and non-standard expansions in the theory of the Boltzmann kinetic equation, in: R.G. Khlebopros (Ed.), Mathematical Modelling in Biology and Chemistry. New Approaches, Nauka Publisher, Novosibirsk, 1991, pp. 69-117 (in Russian).

[39] A.N. Gorban, I.V. Karlin, Quasi-Equilibrium Closure Hierarchies for The Boltzmann Equation, e-print (2003). On-line: arXiv: cond-mat/0305599.

[40] R.B. Bird, C.F. Curtiss, R.C. Armstrong, O. Hassager, Dynamics of Polymer Liquids, second ed., Wiley, New York, 1987.

[41] M. Hütter, I.V. Karlin, H.C. Öttinger, Dynamical mean-field models from a nonequilibrium thermodynamics perspective, Phys. Rev. E 68 (2003) 016115.

[42] V.I. Arnold, A.N. Varchenko, S.M. Gussein-Zade, Singularities of Differentiable Maps, vol. 2, Brickhäuser, Boston [etc.], 1985-1988.

[43] E.M. Lifshitz, L.P. Pitaevskii, Physical kinetics, L.D. Landau, E.M. Lifshitz (Eds.), Course of Theoretical Physics, vol.10, Pergamon Press, Oxford, 1968.

[44] B. Simon, The $P(\Phi)_{2}$ Euclidian (Quantum) Field Theory, Princeton Series in Physics, Princeton University Press, 1974.

[45] B. Holmquist, Moments and cumulants of the multivariative normal distribution, stochastic analysis and applications 6 (1988) 273-278.

[46] A.N. Gorban, I.V. Karlin, V.B. Zmievskii, T.F. Nonnenmacher, Relaxational trajectories: global approximations, Physica A 231 (1996) 648-672.

[47] A.N. Gorban, I.V. Karlin, V.B. Zmievskii, Two-step approximation of space-independent relaxation, Transport Theory Stat. Phys. 28 (3) (1999) 271-296.

[48] P.G. De Gennes, Molecular individualism, Science 276 (5321) (1997) 1999-2000.

[49] Th.T. Perkins, D.E. Smith, S. Chu, Single polymer dynamics in an elongational flow, Science 276 (5321) (1997) 2016-2021.

[50] P. Leduc, Ch. Haber, G. Bao, D. Wirtz, Dynamics of individual flexible polymers in a shear flow, Nature 399 (1999) 564-566. 